Discussion Paper No. 1006

\title{
MULTISTAGE INFORMATION TRANSMISSION WITH VOLUNTARY MONETARY TRANSFER
}

\author{
Hitoshi Sadakane
}

June 2017

The Institute of Social and Economic Research

Osaka University

6-1 Mihogaoka, Ibaraki, Osaka 567-0047, Japan 


\title{
Multistage Information Transmission with Voluntary Monetary Transfer ${ }^{\dagger}$
}

\author{
Hitoshi Sadakane ${ }^{\ddagger}$ \\ Institute of Economic Research, Kyoto University
}

June, 2017

\begin{abstract}
We examine multistage information transmission with voluntary monetary transfer in the framework of Crawford and Sobel (1982). In our model, an informed expert can send messages to an uninformed decision maker more than once, and the uninformed decision maker can pay money to the informed expert voluntarily whenever she receives a message. Our results are that under some conditions (i) the decision maker can obtain more detailed information from the expert than that in the Crawford and Sobel model and (ii) there exists an equilibrium whose outcome Pareto dominates all the equilibrium outcomes in the Crawford and Sobel model. Moreover, we find the upper bound of the receiver's equilibrium payoff, and provide a sufficient condition for it to be approximated by the receiver's payoff under a certain equilibrium.
\end{abstract}

JEL Classification: C72; C73; D82; D83

Keywords: Incomplete information; Cheap talk; Multistage strategic communication; Voluntary monetary transfer

\footnotetext{
${ }^{\dagger}$ I am grateful to Yasuyuki Miyahara for his invaluable support. I also would like to thank Hideo Suehiro, Hideshi Itoh, Hisao Hisamoto, Kohei Kawamura, Shinsuke Kambe, Shintaro Miura, Takashi Shimizu, Takeshi Murooka and seminar participants at Contract Theory Workshop, Contract Theory Workshop summer camp, KIER and ISER for their helpful comments and suggestions. This paper is the 19th ISER-Moriguchi Prize awarded paper. All errors are my own.

¥Institute of Economic Research, Kyoto University, Sakyo-ku, Kyoto 6068501, Japan

E-mail addresses: sadakane@kier.kyoto-u.ac.jp
} 


\section{Introduction}

Private information is transmitted in organizations during the decision-making process. One typical information transmission method is ordinary and informal talk, which we call "cheap talk." Cheap talk itself does not affect the payoffs of parties. However, what it conveys often affects future decision making. Then, parties may transmit their private information strategically.

The seminal analysis of the strategic communication between an informed expert (sender or he) and an uninformed decision maker (receiver or she) was provided by Crawford and Sobel (1982), hereafter CS. In their model, communication is unilateral and one-shot. More precisely, the sender sends a message to the receiver, and then the receiver chooses a project. The major results in the CS model are that the existence of the misalignment of preferences between the sender and the receiver typically prevents full revelation of information and that there exists a partially informative equilibrium when the incentive conflict is not too large.

CS assume that the receiver is passive in the communication process. A number of situations are like this. In other situations, the receiver may participate in the communication process actively, e.g., she may compensate the sender for his messages. The present paper studies multistage communication as an extension of the CS model and investigate how information transmission can be improved through the receiver's active participation in the communication process. In particular, we focus on multistage information transmission with voluntary monetary transfer.

Our communication procedure differs from that of the CS model in two ways. First, the sender can send messages to the receiver unilaterally more than once. We assume that sending messages is costless ${ }^{1}$ and messages are unverifiable. ${ }^{2}$ Second, the receiver can pay money to the sender voluntarily whenever she receives a message from the sender. More specifically, the receiver and sender engage in finite-period communication before the former chooses a project. In each period, the sender sends an unverifiable message to the receiver without cost, and then, the receiver pays money to the sender voluntarily. After this finite-period communication, the receiver chooses a project.

\footnotetext{
${ }^{1}$ Spence (1973) studies a signaling model where a informed party conveys information by taking actions that affect their own utility. In the framework of the CS model, Austen-Smith and Banks (2000), Kartik (2007), and Karamychev and Visser (2016) show that information transmission can be improved when the sender can send a costly message (burn money) to signal information. Relatedly, Kartik et al. (2007) and Kartik (2009) study amendments to the CS model with other means of costly signals such as lying costs. In the present paper, we focus on the receiver's active participation in the communication process. Thus, we assume that the sender cannot send a costly signal.

${ }^{2}$ Seidmann and Winter (1997) and Mathis (2008) study the sender-receiver game where the message sent by the sender is (partially) verifiable, namely the set of available messages depends on the sender's type. They provide the sufficient conditions (the latter provides the necessary and sufficient conditions) for the existence of a fully revealing equilibrium. Forges and Koessler (2008) and Hörner and Skrzypacz (2016) study multistage sender-receiver games with certifiable messages (the long persuasion). The former geometrically characterizes the set of equilibrium payoffs. The latter shows that the sequential revelation of partially informative signals can increase payments to the sender who is trying to sell his information to the receiver.
} 
In the present paper, we show that when the receiver places greater importance on the project than the sender does, (i) the receiver can obtain more detailed information from the expert than in the CS model ${ }^{3}$ and (ii) there exists an equilibrium whose outcome Pareto-dominates all the equilibrium outcomes in the CS model.

We also show that there exists no fully separating equilibrium in our model. This implies that information transmission is limited in our communication procedure. By concentrating on the well-known uniform-quadratic model (i.e., with quadratic preferences regarding the project and a uniform type distribution), we find an upper bound of the receiver's equilibrium payoff and show that this can be approximated by the receiver's payoff under a certain equilibrium when the communication round is high.

Since we assume that messages are unverifiable, the receiver cannot write a contract that specifies the monetary transfer depending on the messages sent by the sender. When the receiver cannot write a contract and communication is one-shot, voluntary monetary transfer does not affect information transmission. Moreover, allowing multiple rounds of unilateral (one-sided) communication in the CS model does not affect the set of equilibria identified by the original model. For details, see Krishna and Morgan (2004). ${ }^{4}$ We show that information transmission can be improved by combining multistage information transmission with voluntary monetary transfer.

The intuition for why the receiver pays money to the sender under the equilibrium in our model is as follows. In any cheap talk game, there always exists a completely uninformative equilibrium (the babbling equilibrium). Therefore, if there is a partially informative equilibrium, then there are multiple equilibria. Thus, the dependence of the selection of the future equilibrium on the receiver's past payment creates an incentive for the receiver to pay money to the sender. Similar to Benoit and Krishna (1985), we use the multiple equilibria in the remaining (original) game to provide an incentive for voluntary payments.

Consider the situation where a (partially) informative equilibrium is played in the future only when the receiver pays a certain amount of money to the sender in the current period. Otherwise, play continues according to the babbling equilibrium. In such a situation, the higher the receiver's importance towards a project than towards monetary transfer, the more she is willing to pay money in the current period to ensure that the uninformative equilibrium would not be chosen in the future.

In our communication procedure, the sender sends a message while paying attention not only to the expected outcome from the project that would be implemented in the future but also to the expected future monetary transfer. This fact means that the possibility of the future monetary transfer affects the sender's incentive regarding sending a message in the current period, implying that the receiver can control the sender's incentive through voluntary monetary transfer to a certain degree.

\footnotetext{
${ }^{3}$ This result means that there exists an equilibrium whose partition has a greater number of elements than that achieved in any equilibrium in the CS model.

${ }^{4}$ Allowing multiple rounds of "bilateral" (face-to-face or two-sided) communication in the CS model could result in equilibria that Pareto-dominate those of the original model. For more details, see Krishna and Morgan (2004).
} 
The intuition for how the receiver controls the sender's incentive through voluntary monetary transfer is simple. In the CS model, given a state of the world, the most desirable project for the sender always differs from that for the receiver. This fact means that in the CS model, excessively detailed information transmission is prevented since the sender has an incentive to deceive the receiver into choosing a project that is more desirable for him. By contrast, in our model, the receiver can weaken this cheating incentive of the sender by paying money to him when he conveys some information that is contrary to the bias.

It is obvious that there always exists an equilibrium under which players waste time in the communication round regardless of its length. For instance, irrespective of the length of the communication round is, there always exists an equilibrium under which the sender sends an informative message to the receiver at most once and the receiver never pays money. The equilibrium partition achieved in such an equilibrium is always achievable in the CS model. It seems that $\mathrm{R}$ does not use T-period communication effectively under these equilibria. In the present paper, we show the benefit of long term communication by demonstrating that under a certain condition, there exists an equilibrium where information is transmitted within an extended period of time.

Dessein (2002) studies the delegation problem. In his model, the receiver chooses whether to communicate with the sender. She then makes a decision herself or fully delegates the decision-making authority to the sender. He shows that the receiver prefers full delegation to communication as long as the incentive conflict is not too large. Since the work of Dessein (2002), the problem of "when players should communicate (or delegate) in an organization" has been the object of extensive study. Moreover, it has been shown that if the receiver can commit herself to a predetermined decision rule, she can always obtain higher ex ante expected payoff than that in any equilibrium in the CS model. For details, see Goltsman et al. (2009). Note that the optimal predetermined decision rule is always no worse than full delegation. Hence, if the receiver can write a contract that determines the decision making ex ante, then communication (without transfer) is not necessary for good decision making.

We compare multistage information transmission with voluntary monetary transfer with the optimal predetermined decision rule induced by Goltsman et al. (2009) to investigate the most effective communication protocol for the receiver. Surprisingly, when the communication round is high, the receiver may obtain a higher ex ante expected payoff than that under optimal arbitration under which players benefit from the "formal contract" that forces them to commit themselves to the predetermined decision rule.

The present paper is organized as follows. Section 2 introduces the model. Section 3 characterizes the equilibria. Section 4 derives the general properties of these equilibria. Section 5 analyzes the uniform-quadratic model and shows the benefits of multistage information transmission with voluntary monetary transfer. In Section 5.1, we show two main results by constructing an equilibrium where information is transmitted within two periods. In Section 5.2, we show the benefit of long-term communication. In Section 5.3, we compare our communication procedure with other communication protocols. In Section 6, we generalize players' payoff functions and the prior probability 
distribution, and show two results that correspond to the results in Section 5.1. Section 7 concludes.

\subsection{Related Literature}

CS study the one-shot unilateral communication game in which the sender sends a costless and unverifiable message about his private information to the receiver, who then makes a decision regarding the project that affects the payoffs of both parties. They obtain a complete characterization of the set of equilibria in their model and show that the existence of the incentive conflict prevents the full revelation of information. In the present paper, we investigate how information transmission can be improved under multistage information transmission with voluntary monetary transfer.

Krishna and Morgan (2008) study an amendment to the CS model by allowing the receiver to write a contract that specifies the monetary transfer depending on the message sent by the sender. They show that full information revelation is feasible but not optimal and they characterize the optimal contract. In their model, there is a crucial assumption that the receiver can commit herself to compensate the sender for his message. We show that in the situation where the communication round has multiple periods, the receiver can control the sender's incentive through voluntary payment, although she cannot contract with the sender.

Our results are closely related to those of Krishna and Morgan (2004). Both their study and our analysis in the present paper investigate how information transmission can be improved through the receiver's active participation in the communication process. Krishna and Morgan (2004) add a long communication protocol to the CS model. They show that if bilateral (face-to-face) communication between the receiver and sender is possible before the sender sends a message about his private information to the receiver, there exists an equilibrium whose outcome Pareto-dominates all the equilibrium outcomes in the CS model. The key factor to their results is that after the sender conveys some information in the face-to-face communication, multiple equilibria exist in the remaining game. The outcome of this face-to-face communication, which could be random, determines which of these equilibria is played in the future. This fact affects what the sender conveys during the face-to-face communication. Therefore, in Krishna and Morgan (2004), the receiver tries to control the sender's incentive by controlling the degree of uncertainty associated with the outcome of the face-to-face communication. By contrast, in our model, the receiver tries to control the sender's incentive directly through voluntary monetary transfers. Moreover, there always exists a completely uninformative (babbling) equilibrium in the CS model. Therefore, the receiver's voluntary monetary transfer is supported by a fear of the sender's punishment of babbling.

Noisy communication leads to improved information transmission (e.g., Krishna and Morgan, 2004; Blume et al., 2007; Goltsman et al., 2009; Ivanov, 2010; Ambrus et al., 2013). ${ }^{5}$ Blume et al. (2007) and Goltsman et al. (2009) characterize the optimal level

\footnotetext{
${ }^{5}$ Aumann and Hart (2003) study a finite simultaneous-move (long conversation) game in which there are two players, with one player better informed than the other. They provide a complete geometrical
} 
of noise (precisely, the latter characterize the optimal device, which they call "optimal mediation," that controls the noise in communication) and provide a least upper bound of the receiver's equilibrium payoff.

In Section 5.1, we compare our communication procedure with optimal mediation and show that when the receiver places greater importance on the project than the sender does, the receiver can obtain a higher ex ante expected payoff than that under optimal noisy communication (mediation).

As shown by Dessein (2002), the receiver prefers delegation to communication as long as the incentive conflict is not too large. In particular, in the uniform-quadratic case of the CS model, the receiver always prefers delegation to communication even when informative communication is feasible. This implies that it is important to investigate when the receiver should communicate with the sender instead of delegating authority. Moreover, Goltsman et al. (2009) tackle the problem of designing a pre-determined decision rule which includes full delegation analyzed by Dessein (2002). They provide a potentially stochastic mechanism in the framework of the CS model: arbitration. Under the arbitration, a neutral third-party (arbitrator) can ask the sender for information and commit to a pre-determined potentially stochastic decision rule. Goltsman et al. (2009) characterize the optimal arbitration mechanism. ${ }^{6}$ Accordingly, we compare our communication procedure with the optimal arbitration and show that the receiver can obtain higher ex ante expected payoff than that under the optimal arbitration when the length of the communication round is high and the incentive conflict is not too large.

In all the studies mentioned above, once the communication phase is over, the receiver chooses a project. By contrast, in the previous studies we mention below, there are multiple rounds of communication and actions. More precisely, in each period, the sender sends a message and then the receiver chooses a project. Hence, the models are different from ours. Golosov et al. (2014) study strategic information transmission in a finitely repeated cheap talk game. Only the sender knows the state of the world, which remains constant through out the game. They show that the sender can condition his message on the receiver's past actions; additionally, the receiver can choose actions that reward the sender for following a path of messages that eventually leads to the full revelation of information. By contrast, there is no fully revealing equilibrium in our model. For truth telling to be incentive compatible, the resulting payment (i.e., the sum of the monetary transfer that the sender receives) must be different for each state. This point means that there must exist a on-the-path history where the receiver pays a positive amount of money to the sender even though she would not obtain additional information after this payment. At such a history, she has no incentive to pay money

characterization of the set of equilibrium payoffs when the state of the world is finite and a long communication is possible. In this study, state space and players' action space must be finite. Therefore, we cannot directly apply the results of this study to our model.

${ }^{6}$ One simple decision rule for the receiver is delegate the sender to make a decision, but possibly to constrain the set of available decisions. This class of mechanisms includes delegation mechanisms analyzed in Holmström (1977), Melumad and Shibano (1991), and Alonso and Matouschek (2008). Goltsman et al. (2009) show that the optimal arbitration mechanism is deterministic as a consequence. This shows that the optimal arbitration includes the optimal delegation mechanism. 
to the sender. Therefore, there is no fully revealing equilibrium.

Kolotilin and $\mathrm{Li}$ (2016) investigate the optimal relational contracts in an infinitely repeated cheap talk game. In contrast to our study, the sender's private information is not persistent in their model. Additionally, in each period, both the sender and the receiver can pay money to each other. In their model, there are equilibria where the sender always reveals his private information completely since he can send a costly signal by paying money to the receiver. They show that full separation can be attained in the equilibrium, whereas partial or complete pooling is optimal if preferences are divergent. Moreover, they allow the sender to pay money to the receiver. Therefore, the optimal relational contract that maximizes social welfare in their model differs from effective communication in our model.

\section{Model}

There are two players, a sender $(S)$ and a receiver $(R)$. $R$ has the authority to choose a project $y \in Y \equiv \mathbb{R}_{+}$, but the outcome produced by project $y$ depends on $S^{\prime}$ s private information, $\theta$, which is distributed according to a differentiable distribution function $G(\theta)$, with density $g(\theta)$, over $\Theta \equiv[0,1]$.

Before $R$ chooses a project, $R$ and $S$ engage in $T$-period communication. Each period consists of two-stage, stage 1 and stage 2 . Stage 1 is the report stage, where $S$ sends a costless and unverifiable message to $R$. Let $M \equiv[0,1]$ be $S$ 's message space. We denote by $m_{t}$ a message sent by $S$ at the report stage in period $t$. Stage 2 is the transfer stage, where $R$ voluntarily pays money to $S$. Let $W \equiv \mathbb{R}_{+}$be the set of the amount of payment possible for $R$. We denote by $w_{t}$ an amount of payment, which $R$ pays to $S$ at the transfer stage in period $t$.

After $T$-period communication, we proceed to period $T+1$, where $R$ chooses a project and the game ends. Let $w$ be a sequence of transfers, $w \equiv\left(w_{1}, \ldots, w_{T}\right) \in W^{T}$. We define $R^{\prime}$ s payoff function $U^{R}: Y \times \Theta \times W^{T} \rightarrow \mathbb{R}$ as follows:

$$
U^{R}(y, \theta, w) \equiv r \cdot u^{R}(y, \theta)-\sum_{t=1}^{T} w_{t}
$$

where $r$ is a positive constant which represents a coefficient of loss aversion with respect to a project she chose.

We define $S^{\prime}$ s payoff function $U^{S}: Y \times \Theta \times W^{T} \rightarrow \mathbb{R}$ as follows:

$$
U^{S}(y, \theta, w) \equiv s \cdot u^{S}(y, \theta, b)+\sum_{t=1}^{T} w_{t}
$$

where $s$ is a positive constant which measures the relative importance between monetary transfer and the utility from a project. Parameter $b>0$ represents a "bias", which measures how much $S^{\prime}$ s interest differs from $R^{\prime}$ s.

Here, $r \cdot u^{R}(y, \theta)$ and $s \cdot u^{S}(y, \theta, b)$ denote utilities from project $y$ for $R$ and $S$, respectively. We assume that for $i \in\{R, S\}$, the function $u^{i}$ is twice-continuously differentiable and satisfies assumptions that for each $\theta$ and $b, u_{11}^{i}<0$ and $u_{12}^{i}>0$, and $u_{1}^{i}=0$ for 
some $y$. Since $u_{1}^{i}=0$ for some $y$ and $u_{11}^{i}<0$, for each given $(\theta, b)$ there exists a unique maximizing project: $y^{R}(\theta)=\arg \max _{y} u^{R}(y, \theta)$ and $y^{S}(\theta, b)=\arg \max _{y} u^{S}(y, \theta, b)$. We assume that $u^{R}(y, \theta) \equiv u^{S}(y, \theta, 0)$ and $u_{13}^{S}>0$ everywhere. Since $u_{13}^{S}>0$ and $b>0$, we have $y^{R}(\theta)<y^{S}(\theta, b)$ for each $\theta$. Constants $r>0$ and $s>0$ are scalar parameters. The former measures how strongly $R$ prefers the project, $y=y^{R}(\theta)$, which is the most desirable for $R$. The latter measures how strongly $S$ prefers the project, $y=y^{S}(\theta, b)$, which is the most desirable for $S$. $\sum_{t=1}^{T} w_{t}$ is the sum of monetary transfers. In what follows, we denote by $\Gamma(b, s, r, T)$ our $T$-period communication game.

\subsection{History and Strategies}

A (public) history $h^{(t, j)}$ is defined to be a sequence of players' past actions realized until the beginning of the stage $j$ in period $t$.

$$
h^{(t, j)} \equiv\left\{\begin{array}{l}
\left(m_{1}, w_{1}, \ldots, m_{t-1}, w_{t-1}\right) \quad \text { if } j=1, \\
\left(m_{1}, w_{1}, \ldots, m_{t-1}, w_{t-1}, m_{t}\right) \quad \text { if } j=2 .
\end{array}\right.
$$

A (public) history $h^{T+1}$ is defined to be a sequence of players' past actions realized until the beginning of the period $T+1$, where $R$ chooses a project.

$$
h^{T+1} \equiv\left(m_{1}, w_{1}, \ldots, m_{T}, w_{T}\right) .
$$

Let $H^{(t, j)}$ and $H^{T+1}$ be the set of $h^{(t, j)}$ and $h^{T+1}$, respectively. We assume that $H^{(1,1)}$ is a singleton set $\{\phi\}$. Let us denote the set of all histories at stage $j$ by $\mathcal{H}^{j} \equiv \bigcup_{t=1}^{T} H^{(t, j)}$.

Let $h_{\theta}^{(t, 1)} \in \Theta \times H^{(t, 1)} \equiv H_{\Theta}^{(t, 1)}$ be an $S^{\prime}$ s private history at stage 1 in period $t$. Let $\mathcal{H}_{\Theta}^{1}$ be the set of all $S^{\prime}$ s private histories, $\mathcal{H}_{\Theta}^{1} \equiv \Theta \times \mathcal{H}^{1}$. An $S^{\prime}$ s behavior strategy, $\sigma: \mathcal{H}_{\Theta}^{1} \rightarrow \Delta M$, specifies the probability distribution of messages $S$ of type $\theta$ sends at stage 1 in period $t^{7}$

An $R^{\prime}$ s pure strategy is s measurable function $\rho: \mathcal{H}^{2} \cup H^{T+1} \rightarrow \mathbb{R}_{+}$, specifies the amount of payment she pays at stage 2 in the communication phase and the project she chooses in period $T+1$. Note that $\rho\left(h^{(t, 2)}\right) \in W$, and $\rho\left(h^{T+1}\right) \in Y^{8}$. A belief system, $f: \mathcal{H}^{2} \cup H^{T+1} \rightarrow \Delta \Theta$, specifies the $R^{\prime}$ 's belief about $S^{\prime}$ s types at a history $h \in \mathcal{H}^{2} \cup H^{T+1}$.

\section{Equilibrium}

Let $\mathbb{H} \equiv \Theta \times M_{1} \times W_{1} \times \cdots \times M_{T} \times W_{T} \times Y$ be the set of sequences of the realized state and players' actions, $\left(\theta, m_{1}, w_{1}, \ldots, m_{T}, w_{T}, y\right) .{ }^{9}$ Let $\mathbb{B}(\mathbb{H})$ be the Borel algebra on $\mathbb{H} .{ }^{10}$

\footnotetext{
${ }^{7}$ We denote by $\mathbb{B}(X)$ the Borel algebra on a set $X$. An $S^{\prime}$ 's behavior strategy is a function $\sigma: \mathbb{B}(M) \times \mathcal{H}_{\Theta}^{1} \rightarrow$ $[0,1]$ with the following two properties: (1) For every $\tilde{M} \in \mathbb{B}(M)$, the function $\sigma(\tilde{M}, \cdot): \mathcal{H}_{\Theta}^{1} \rightarrow[0,1]$ is measurable. (2) For every $h_{\theta}^{(t, 1)} \in \mathcal{H}_{\Theta}^{1}$, the function $\sigma\left(\cdot, h_{\theta}^{(t, 1)}\right): \tilde{M} \rightarrow[0,1]$ is a probability measure. The definition of $\sigma$ is originated from Milgrom and Weber (1985).

${ }^{8}$ Due to the strict concavity of $R^{\prime}$ s preference over projects, she never mixes between projects in period $T+1$.

${ }^{9}$ In order to avoid confusion, we add a time operator to the players' action space.

${ }^{10}$ Suppose that $\mathbb{R}^{k}$ and $\mathbb{R}^{l}$ are equipped with their Borel algebras $\mathbb{B}\left(\mathbb{R}^{k}\right)$ and $\mathbb{B}\left(\mathbb{R}^{l}\right)$, respectively, and let $\mathbb{R}^{k+l}=\mathbb{R}^{k} \times \mathbb{R}^{l}$. Then, $\mathbb{B}\left(\mathbb{R}^{k+l}\right)=\mathbb{B}\left(\mathbb{R}^{k}\right) \otimes\left(\mathbb{R}^{l}\right)$. Hence, $\mathbb{B}(\Theta \times \cdots \times Y) \equiv \mathbb{B}(\Theta) \otimes \mathbb{B}\left(M_{1}\right) \otimes \cdots \otimes \mathbb{B}\left(W_{T}\right) \otimes \mathbb{B}(Y)$
} 
Given a strategy profile and a prior distribution, $((\sigma, \rho), G)$, a probability measure $\mathbb{P}$ on the measurable space $(\mathbb{H}, \mathbb{B}(\mathbb{H}))$ is uniquely determined. For details, see Appendix L. Given $h \in \mathbb{H}$, the values of players' payoffs, both $U^{R}$ and $U^{S}$, are uniquely derived, respectively. Moreover, the functions $U^{R}: \mathbb{H} \rightarrow \mathbb{R}$ and $U^{S}: \mathbb{H} \rightarrow \mathbb{R}$ are measurable. Therefore, the players' ex ante expected payoffs $\int_{h \in \mathbb{H}} U^{R}(h) \mathbb{P}(d h)$ and $\int_{h \in \mathbb{H}} U^{S}(h) \mathbb{P}(d h)$ are well-defined, respectively.

Fix an $S^{\prime}$ s private history at stage 1 in period $t$ as $h_{\theta}^{(t, 1)}$. We denote by $V^{S}\left(\sigma, \rho \mid h_{\theta}^{(t, 1)}, m_{t}\right)$ the $S^{\prime}$ s continuation payoff after sending $m_{t}$ at $h_{\theta}^{(t, 1)}$. Fix a public history at stage 2 in period $t$ as $h^{(t, 2)}$ and an $R^{\prime}$ s payment $w_{t}$. Let $V^{R}\left((\sigma, \rho), f \mid h^{(t, 2)}, w_{t}\right)$ be the $R^{\prime} s$ continuation payoff after paying $w_{t}$ at this history.

We shall analyze (weak) perfect Bayesian equilibria in $\Gamma(b, s, r, T)$ : both players' strategies must maximize their expected payoffs after all histories, and the system of beliefs $f$ must be consistent with the regular conditional probability derived from $((\sigma, \rho), f)$ and $G^{11}$

Definition 1. A strategy profile $(\sigma, \rho)$ and a belief system $f$ constitute a perfect Bayesian equilibrium if the following conditions hold. For any $t \in\{1, \ldots, T\}$,

1. for any $h_{\theta}^{(t, 1)} \in H_{\Theta}^{(t, 1)}$ and $m_{t} \in \operatorname{supp}\left\{\sigma\left(\cdot \mid h_{\theta}^{(t, 1)}\right)\right\}$,

$$
m_{t} \in \arg \max _{m_{t}^{\prime}} V^{S}\left(\sigma, \rho \mid h_{\theta}^{(t, 1)}, m_{t}^{\prime}\right),
$$

2. for any $h^{(t, 2)} \in H^{(t, 2)}$,

$$
\rho\left(h^{(t, 2)}\right) \in \arg \max _{w_{t}^{\prime}}\left\{V^{R}\left((\sigma, \rho), f \mid h^{(t, 2)}, w_{t}^{\prime}\right)-w_{t}^{\prime}\right\},
$$

3. for any $h^{T+1} \in H^{T+1}$,

$$
\rho\left(h^{T+1}\right) \in \arg \max _{y^{\prime}} r \int u^{R}\left(y^{\prime}, \theta\right) f\left(d \theta \mid h^{T+1}\right),
$$

4. the belief system $f$ is consistent with $(\sigma, \rho) .{ }^{12}$

Hereafter, we call a perfect Bayesian equilibrium simply equilibrium. Suppose that $((\sigma, \rho), f)$ be an equilibrium. At any payment stage history $h^{(t, 2)}, R$ does not obtain additional information about $S$ 's type from her own action $w_{t}$. Therefore, we require that at any $h^{(t, 2)}$, any deviation by $R$ from $\rho\left(h^{(t, 2)}\right)$ does not affect the beliefs she uses as the basis for belief-updating.

\section{Some Properties of Equilibria}

In what follows, we derive some properties of equilibria.

\footnotetext{
${ }^{11} \operatorname{In} \Gamma(b, s, r, T)$, there always exists an equilibrium that is essentially equivalent to a perfect Bayesian equilibrium in the CS model. Hence, in this paper we do not prove the existence theorem.

${ }^{12}$ For details, see Appendix M.
} 


\subsection{Relation to the CS Model}

First, we discuss the relation between the equilibria in the CS model and those in $\Gamma(b, s, r, T)$. Since $R$ cannot obtain additional information about $\theta$ after stage 2 in period $T$, she has no incentive to choose $w^{T}>0$. Therefore, $w^{T}$ must be equal to 0 in any equilibrium. Consequently, $\Gamma(b, s, r, 1)$ is contained in the CS model, and we call it the model with one-shot information transmission. CS have shown that under one-shot information transmission, for every $b>0$, there exists a positive integer $\tilde{n}(b)$ such that, for every $n \in\{1, \ldots, \tilde{n}(b)\}$, there exists an equilibrium with a $n$-element partition, $\left\{\left[a_{n}, a_{n-1}\right),\left[a_{n-1}, a_{n-2}\right), \ldots\left[a_{1}, a_{0}\right]\right\}$. Under this equilibrium, $S^{\prime}$ s type $\theta \in\left[a_{i+1}, a_{i}\right)$ reports that his type belongs to this interval, and after receiving the message that " $\theta$ belongs to $\left[a_{i+1}, a_{i}\right)^{\prime \prime}, R$ chooses the project $\bar{y}\left(a_{i+1}, a_{i}\right) \equiv \arg \max _{y} \int_{a_{i+1}}^{a_{i}} u^{R}(y, \theta) g(\theta) d \theta$. By convention, we define $\bar{y}\left(a_{1}, a_{0}\right)=y^{*}\left(a_{0}\right)$ if $a_{1}=a_{0}$. Since $u^{R}$ is strictly concave, $\bar{y}\left(a_{i+1}, a_{i}\right)$ is uniquely determined. Moreover, since $u_{12}^{R}>0, \bar{y}\left(a_{i+1}, a_{i}\right)$ is strictly increasing in both of its arguments. Therefore, it must be satisfied that: for $i=1, \ldots, n-1$,

$$
\begin{aligned}
& s \cdot u^{S}\left(\bar{y}\left(a_{i+1}, a_{i}\right), a_{i}, b\right)-s \cdot u^{S}\left(\bar{y}\left(a_{i}, a_{i-1}\right), a_{i}, b\right)=0 \\
& a_{n}=0 \\
& a_{0}=1 .
\end{aligned}
$$

We shall call a sequence $a \equiv\left\{a_{0}, \ldots, a_{n}\right\}$ is a (backward) solution of (1) if $a$ satisfies (1)-(3). We shall impose the following monotonicity condition on a solution of (1).

Condition M. If $a^{\prime}$ and $a^{\prime \prime}$ are two solutions of (1) with $a_{0}^{\prime}=a_{0}^{\prime \prime}$ and $a_{1}^{\prime}>a_{1}^{\prime \prime}$, then $a_{i}^{\prime} \geq a_{i}^{\prime \prime}$ for all $i \geq 2$.

This condition is met by the uniform-quadratic case: $s \cdot u^{S}(y, \theta, b) \equiv-s(y-(\theta+b))^{2}$, $r \cdot u^{R}(y, \theta) \equiv-r(y-\theta)^{2}$, and $G(\theta)$ is uniform distribution over [0,1]. CS have shown that Condition $\mathrm{M}$ also holds for more general specifications.

Suppose a strategy profile under which $S$ sends an informative message only at stage 1 in period 1 , and $R$ pays nothing to $S$ at any payment stage. Obviously, if both $S$ 's behavior regarding sending $m_{1}$ and $R$ 's behavior regarding choosing $y$ depending on $m_{1}$ are the same as an equilibrium in the CS model, then this strategy profile constitutes an equilibrium in $\Gamma(b, s, r, T)$. Therefore, we immediately have the following Fact 1 .

Fact 1. Any equilibrium partition achieved in the CS model can be achieved under an equilibrium in $\Gamma(b, s, r, T)$.

\subsection{Relation to Direct Contract}

In this section, we first characterize the relation between equilibria in $\Gamma(b, s, r, T)$ and those in a case where $R$ can write a contract that specifies the transfer as functions of messages sent by $S$.

Fix an equilibrium $\xi=((\sigma, \rho), f)$. Let $\mu_{\xi}: \Theta \rightarrow \Delta\left(M^{T}\right)$ be a probability distribution induced by $(\sigma, \rho)$ over $M^{T}$. When a sequence of messages $\mathbf{m} \in M^{T}$ is given, a sequence of payments $\mathbf{w} \in W^{T}$ and a project $y$ are induced from $\rho$, respectively. Let $\omega_{\xi}: M^{T} \rightarrow W^{T}$ and $y_{\xi}: M^{T} \rightarrow Y$ be the functions induced by $\rho$, respectively. 
Now, consider the case where $R$ can contract $\omega_{\xi}$ with $S$. By the construction of $\omega_{\xi}: M^{T} \rightarrow W^{T}$, under this indirect contract $\left(M^{T}, \omega_{\xi}\right)$, the strategy profile $\left(\mu_{\xi}, y_{\xi}\right)$ constitutes an perfect Bayesian equilibrium that is outcome equivalent to $\xi$ in the sense that both of this equilibrium and $\xi$ induce the same probability distribution over $W^{T} \times Y$ for any $\theta$.

Next, we discuss the relation between equilibria under this indirect contract $\left(M^{T}, \omega_{\xi}\right)$ and those under a direct contract where $R$ can write a contract that specifies the transfer as functions of the direct message $m \in \Theta$ sent by $S$. Let $(\Theta, \omega)$ be a direct contract under which $S$ reports $\theta \in \Theta$ and $R$ pays $\omega(\theta)$ for $S$. Let $y: \Theta \rightarrow Y$ be the $R$ 's strategy under the direct contract $(\Theta, \omega)$. By the application of the result of Krishna and Morgan (2008), ${ }^{13}$ we immediately have the following Fact 2.

Fact 2. Consider an equilibrium under $\left(M^{T}, \omega_{\xi}\right)$. There exists a direct contract $(\Theta, \omega)$ under which there exists a pure strategy equilibrium which is outcome equivalent to the given equilibrium under $\left(M^{T}, \omega_{\xi}\right)$.

Finally, we characterize the relation between equilibria in $\Gamma(b, s, r, T)$ and those under a direct contract $(\Theta, \omega)$. The following Proposition 1 shows that given an equilibrium $\xi$ in $\Gamma(b, s, r, T)$, there exists an equilibrium of a direct contract which is outcome equivalent in the sense that it results in the same projects and transfer as in the original equilibrium for almost every state.

Proposition 1. Fix an equilibrium $\xi$ in $\Gamma(b, s, r, T)$. There exists a direct contract $(\Theta, \omega)$ under which there exists a pure strategy equilibrium which is outcome equivalent to $\xi$.

In the indirect contract cases, $\omega_{\xi}(\mathbf{m})$ specifies a sequence of payments, $w_{1}(\mathbf{m}), \ldots$, $w_{T}(\mathbf{m})$ ), dependently on $\mathbf{m}$. In the direct contract case, $\omega(\theta)$ specifies the resulting transfer dependently on $\theta$. Fact 2 shows that for almost every $\theta$ and for any $\mathbf{m}, \mathbf{m}^{\prime} \in$ $\operatorname{supp} \mu_{\xi}(\cdot \mid \theta)$, it must be satisfied that $\sum_{t=1}^{T} w_{t}(\mathbf{m})=\sum_{t=1}^{T} w_{t}\left(\mathbf{m}^{\prime}\right)=\omega(\theta)$ and the induced projects is same, $y_{\xi}(\mathbf{m})=y_{\xi}\left(\mathbf{m}^{\prime}\right)=y(\theta)$. This means that there exists a direct contract $(\Theta, \omega)$ under which there exists a pure strategy equilibrium which is outcome equivalent to any equilibrium in $\Gamma(b, s, r, T)$.

\subsection{Partition Equilibrium}

As is the case in the CS model, all the equilibria in $\Gamma(b, s, r, T)$ are interval partitional, that is, all the equilibria are partition equilibria.

Definition 2 (Partition Equilibrium). Fix an equilibrium $\xi$ in $\Gamma(b, s, r, T)$. Consider a pure strategy equilibrium, under a direct $\operatorname{contract}(\Theta, \omega)$, which is outcome equivalent to $\xi$. If there exists a family of sets $\left\{\mathcal{I}_{\lambda}\right\}_{\lambda \in \Lambda}$ over $\Theta$ such that

1. $\left\{\mathcal{I}_{\lambda}\right\}_{\lambda \in \Lambda}$ constitutes an interval partition ${ }^{14}$ over $\Theta$,

2. $y(\theta)=y\left(\theta^{\prime}\right)$ for all $\theta, \theta^{\prime} \in \mathcal{I}_{\lambda}$,

\footnotetext{
${ }^{13}$ For details, see Proposition 2 in Krishna and Morgan (2008).

${ }^{14}$ For all $\lambda \neq \lambda^{\prime}, \mathcal{I}_{\lambda} \cap \mathcal{I}_{\lambda^{\prime}}=\emptyset$. For all $\lambda \in \Lambda, \mathcal{I}_{\lambda}$ is convex, and $\bigcup_{\lambda \in \Lambda} \mathcal{I}_{\lambda}=\Theta$.
} 
3. if $\lambda \neq \lambda^{\prime}$, then $y(\theta) \neq y\left(\theta^{\prime}\right)$ for all $\theta \in \mathcal{I}_{\lambda}$ and $\theta^{\prime} \in \mathcal{I}_{\lambda^{\prime}}$, then

we call $\xi$ partition equilibrium, and $\left\{\mathcal{I}_{\lambda}\right\}_{\lambda \in \Lambda}$ equilibrium partition.

We show the following Proposition 2.

Proposition 2. Any equilibrium under a direct contract $(\Theta, \omega)$ is partition equilibrium.

The proof of Proposition 2 can be found in Appendix A. The above Proposition 1 implies that any equilibrium outcome in $\Gamma(b, s, r, T)$ is also achieved in equilibrium under a corresponding direct contract. Therefore, Proposition 2 means that all equilibria in $\Gamma(b, s, r, T)$ are partition equilibria. The following Proposition 3 shows that there is no fully separating equilibria in $\Gamma(b, s, r, T)$.

Proposition 3. There exists no fully separating equilibrium in $\Gamma(b, s, r, T)$.

The proof of Proposition 3 can be found in Appendix B. In Krishna and Morgan (2008), $R$ can commit herself to compensating for $S$ 's message. Therefore, fully separating equilibria (full revelation contracts) are always feasible in their model. By contrast, in our model, since $R$ cannot commit herself to compensating for $S^{\prime}$ s message, she pays money to $S$ only when paying money is optimal for her. For truth telling to be incentive compatible, it must be satisfied that the resulting sum of monetary transfer that $S$ receives is different for each $\theta \in \Theta$. Precisely, that must be strictly decreasing in $\theta$. This means that $R$ reaches almost surely a history where she pays a certain amount of money to $S$ even though she does not obtain additional information in the future. Therefore, $R$ has no incentive to pay money to $S$ at such a history. This is the reason why there exists no fully separating equilibrium.

\section{The Uniform-quadratic Case}

As noted earlier, it is hard to obtain the full characterization of equilibria. In what follows, we take a small step in that direction, concentrating on the well-known uniformquadratic case: $r \cdot u^{R}(y, \theta)=-r(y-\theta)^{2}, s \cdot u^{S}(y, \theta, b)=-s(y-(\theta+b))^{2}$, and $G(\theta)$ is a uniform distribution over $\Theta$.

\subsection{Two-period Information Elicitation}

As a starting point, we construct an equilibrium where information is transmitted within two period and $R$ pays a positive amount of money to $S$ on the equilibrium path. By constructing such an equilibrium, we show two results. One is that if $T \geq 2$ and $r$ is large relative to $s, R$ can obtain more detailed information in our model than that in a model with one-shot information transmission. The other is that if $T \geq 2$ and $r$ is large relative to $s$, there exists an equilibrium whose outcome ex ante Pareto-dominates all the equilibrium outcomes in a model with one-shot information transmission. In Section 6, we show these two results under more general setting.

To simplify the analysis, we suppose that $b \in(1 / 12,1 / 4)$. Then, there are two equilibria in a model with one-shot information transmission. One is the uninformative 
equilibrium: $a_{0}=1$ and $a_{1}=0$. The other is a partially informative equilibrium: $a_{0}=1, a_{1}=1 / 2-2 b$ and $a_{2}=0$. CS have shown that both $S$ and $R$ prefer the partially informative equilibrium to the uninformative equilibrium. Under the partially informative equilibrium, the ex ante expected payoff of $R$ is $-r\left(1 / 48+b^{2}\right)$ whereas the ex ante expected payoff of $S$ is $-s\left(1 / 48+b^{2}\right)-s b^{2}$.

Now, we show the following Proposition 4 which establishes that in multistage information transmission with voluntary monetary transfer, there exists an equilibrium whose partition has more steps than that in a model with one-shot information transmission.

Proposition 4. Fix $b \in(1 / 12,1 / 4)$. If $s / r<(1-4 b) /(1+12 b)$, then there exists a perfect Bayesian equilibrium with a 3-element partition.

Proof of Proposition 4. To prove this Proposition, we construct a 3-element partition equilibrium under which $S$ conveys $\theta<a_{1}$ or not in period 1 . After conveying $\theta<a_{1}$, then $S$ conveys in period 2 whether $\theta$ belongs to $\left[0, a_{2}\right)$ or $\left[a_{2}, a_{1}\right)$.

More precisely, we consider a strategy profile under which the information is transmitted in the following steps. At stage 1 in period $1, S$ of type $\theta<a_{1}$ sends a message $m_{1}$ randomly according to a uniform distribution over $\left[0, a_{1}\right)$, and $S$ of type $\theta \geq a_{1}$ sends a message $m_{1}$ randomly according to a uniform distribution over $\left[a_{1}, 1\right]$. If $R$ receives $m_{1}<a_{1}$ at stage 1 in period 1 , then she pays $w_{1}=w$ to $S$. Otherwise, she pays nothing to $S$ at stage 2 inperiod 1 . At stage 1 in period 2, if $m_{1}<a_{1}$ and $w_{1} \geq w$, then $S$ of type $\theta<a_{2}$ sends a message $m_{2}$ randomly according to a uniform distribution over [0, $a_{2}$ ), and $S$ of type $\theta \geq a_{2}$ sends a message $m_{2}$ randomly according to a uniform distribution over $\left[a_{2}, 1\right]$. Otherwise, any type of $S$ sends a message $m_{2}$ randomly according to a uniform distribution over [0,1]. In period $t \geq 2 R$ pays nothing to $S$. In period $t \geq 3, S$ always sends babbling message.

Under the above strategy profile, $R$ eventually learns whether $\theta$ belongs to $\left[0, a_{2}\right)$, $\left[a_{2}, a_{1}\right),\left[a_{1}, 1\right]$ or $\left.\left[0, a_{1}\right)\right\}$. Note that $\left[0, a_{1}\right)$ is the support of $R^{\prime}$ s belief at off-the-path $h^{3}$ : $m_{1}<a_{1}, w_{1}<w$ and $m_{2} \in[0,1]$.

At $h^{T+1}$ where $m_{1} \geq a_{1}$, since $R$ believes $\theta$ is uniformly distributed over $\left[a_{1}, 1\right]$, optimal project for $R$ is $y_{1}=\left(a_{1}+1\right) / 2$. At $h^{T+1}$ where $m_{1}<a_{1}, w_{1} \geq w$, and $m_{2} \geq a_{2}$, since $R$ believes $\theta$ is uniformly distributed over $\left[a_{2}, a_{1}\right)$, optimal project for $R$ is $y_{2}=\left(a_{2}+a_{1}\right) / 2$. At $h^{T+1}$ where $m_{1}<a_{1}, w_{1} \geq w$, and $m_{2}<a_{2}$, since $R$ believes $\theta$ is uniformly distributed over $\left[0, a_{2}\right)$, optimal project for $R$ is $y_{3}=a_{2} / 2$. At $h^{T+1}$ where $m_{1}<a_{1}$ and $w_{1}<w$, since $R$ believes $\theta$ is uniformly distributed over $\left[0, a_{1}\right)$, optimal project for $R$ is $\tilde{y}=a_{1} / 2$.

Figure 1 illustrates the equilibrium strategy. In period $t \geq 2, R$ always pays nothing to $S$. This implies that the partition $\left\{\left[0, a_{2}\right),\left[a_{2}, a_{1}\right)\right\}$ must coincide with the 2-element equilibrium partition achieved in a model with one shot information transmission where $\theta$ is drawn from the uniform distribution over $\left[0, a_{1}\right)$. By Crawford and Sobel, it must be satisfied that

$$
a_{2}=a_{1} / 2-2 b .
$$

Since we now construct a 3-element partition equilibrium where $0<a_{2}<a_{1}<1$, it must be satisfied that $a_{2}>0$. Hence, it must be satisfied that $a_{1}>4 b$. 


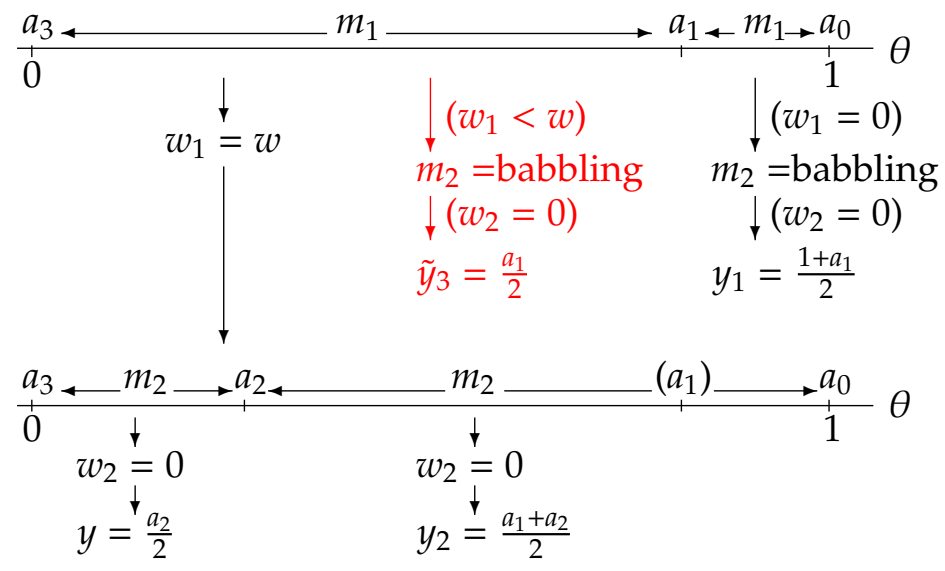

Figure 1: Equilibrium Strategy

The key of our idea is that information elicitation is divided into two steps. After receiving the message $m_{1}<a_{1}$ in period 1 , there are two "cheap talk" equilibria in the remaining game. One is the completely uninformative equilibrium, that is, babbling equilibrium whose partition is $\left\{\left[0, a_{1}\right)\right\}$. The other is partially informative equilibrium, that is, 2-element partition equilibrium whose partition is $\left\{\left[0, a_{2}\right),\left[a_{2}, a_{1}\right)\right\}$. After receiving the message $m_{1}<a_{1}, R$ pays $w_{1}=w$ to $S$ so that the babbling equilibrium would not be chosen in period 2. Furthermore, this $R^{\prime}$ s "message contingent payment" has an impact on $S^{\prime}$ s incentive.

In what follows, we make sure that by taking $a_{1}, a_{2}$ and $w$ suitably, we can constitute an equilibrium where $S$ and $R$ follow the above strategy profile. Under the above strategy profile, $S$ of type $\theta \in\left(a_{i}, a_{i-1}\right)$ sends messages so that $y_{i}$ would be chosen by $R$. Hence, the payoff of $S^{\prime}$ s type $\theta \in\left(a_{i}, a_{i-1}\right)$ is derived as follows:

$$
\begin{aligned}
& -s\left(y_{3}-(\theta+b)\right)^{2}+w \text { for } \theta \in\left[0, a_{2}\right) \\
& -s\left(y_{2}-(\theta+b)\right)^{2}+w \text { for } \theta \in\left[a_{2}, a_{1}\right) \\
& -s\left(y_{1}-(\theta+b)\right)^{2} \text { for } \theta \in\left[a_{1}, 1\right] .
\end{aligned}
$$

Since we suppose that $a_{2}=a_{1} / 2-2 b$, we have

$$
\begin{aligned}
& -s\left(y_{3}-(\theta+b)\right)^{2}>-s\left(y_{2}-(\theta+b)\right)^{2} \text { for } \theta \in\left[0, a_{2}\right) ; \\
& -s\left(y_{3}-(\theta+b)\right)^{2}<-s\left(y_{2}-(\theta+b)\right)^{2} \text { for } \theta \in\left(a_{2}, 1\right] \\
& -s\left(y_{3}-(\theta+b)\right)^{2}=-s\left(y_{2}-(\theta+b)\right)^{2} \text { for } \theta=a_{2} .
\end{aligned}
$$

This implies that at stage 1 in period 2 where $m_{1}<a_{1}$ and $w_{1} \geq w, S$ has no incentive to deviate from given strategy.

At stage 1 in period 2 where $m_{1}<a_{1}$ and $w_{1}<w$, and at stage 1 in period 2 where $m_{1} \geq a_{1}, S$ always sends a babbling message. Therefore, $S$ has no incentive to deviate 
at such a history. The same can be said in period $t \geq 3$. Hence, we can conclude that $S$ has no incentive to deviate in period $t \geq 2$ when $a_{2}=a_{1} / 2-2 b$.

At stage 1 in period 1 , if $S$ of type $\theta$ sends $m_{1} \geq a_{1}$, then he obtains $-s\left(y_{1}-(\theta+b)\right)^{2}$. Otherwise, $S$ of type $\theta \geq a_{2}$ obtains $-s\left(y_{2}-(\theta+b)\right)^{2}+w$, and $S$ of type $\theta<a_{2}$ obtains $-s\left(y_{3}-(\theta+b)\right)^{2}+w$. If it is satisfied that

$$
\begin{aligned}
& -s\left(y_{1}-\left(a_{1}+b\right)\right)^{2}=-s\left(y_{2}-\left(a_{1}+b\right)\right)^{2}+w, \text { then } \\
& -s\left(y_{1}-(\theta+b)\right)^{2} \geq \max _{j \in\{1,2\}}\left\{-s\left(y_{j+1}-(\theta+b)\right)^{2}+w\right\} \text { for } \theta \geq a_{1} ; \\
& -s\left(y_{j+1}-(\theta+b)\right)^{2}+w>-s\left(y_{1}-(\theta+b)\right)^{2} \quad \text { for } i \geq 1 \text { and } \theta \in\left[\hat{a}_{i+1}, \hat{a}_{i}\right) .
\end{aligned}
$$

When (6) and (7) hold, $S$ has no incentive to a deviation at stage 1 in period 1 . The following Figure 2 illustrates (6) and (7).

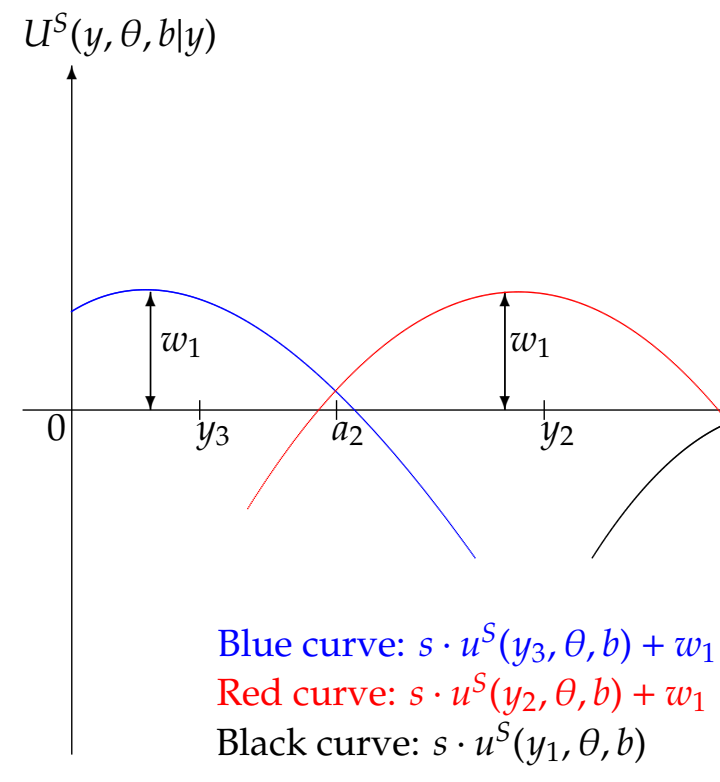

Figure 2: The sender's payoff on the equilibrium path

By the equation (5), we have

$$
w=w\left(a_{1}\right) \equiv s\left[3\left(1 / 2+b-a_{1} / 4\right)\left(a_{1} / 4+b-3 / 2\right)\right] .
$$

Since $w\left(a_{1}\right)$ is strictly increasing in $a_{1} \in[4 b, 1]$, we have an inverse function of $w(\cdot)$ such that $w^{-1}(w) \equiv a_{1}(w)$ is strictly increasing in $w \in[w(4 b), w(1)]$. Since, moreover, we suppose that $b \in(1 / 12,1 / 4)$, it is satisfied that $w(4 b)=s(12 b-1) / 4>0$. Note that $a_{1}(w)=\frac{2}{3}\left\{2-\sqrt{(1+6 b)^{2}-12 w / s}\right\}$ and $a_{1}(w) \in(4 b, 1)$ when $w \in(w(4 b), w(1))$.

Summarizing the above, we can conclude that $S$ has no incentive to a deviation 
when boundaries of partition satisfy the following conditions:

$$
a_{i}(w) \equiv\left\{\begin{array}{l}
1 \text { for } i=0 \\
\frac{2}{3}\left\{2-\sqrt{(1+6 b)^{2}-12 w / s}\right\} \text { for } i=1 \\
\frac{1}{3}\left\{2-\sqrt{(1+6 b)^{2}-12 w / s}\right\}-2 b \text { for } i=2 \\
0 \text { for } i=3
\end{array}\right.
$$

where $w \in(w(4 b), w(1))$.

At any $h^{(t, 2)}, R$ has no incentive to increase the amount of payment because that does not affect $S$ 's behavior. Therefore, we have only to make sure that paying $w$ is optimal for $R$ at stage 2 in period 1 where $m_{1}<a_{1}$.

At stage 2 in period 1 where $m_{1}<a_{1}$, if $R$ pays $w_{1} \geq w$, then she obtains $u^{*}\left(w_{1}\right)$ :

$$
\begin{aligned}
u^{*}\left(w_{1}\right) & =-w_{1}-\frac{1}{a_{1}} \sum_{i=1}^{2} \int_{a_{i+1}}^{a_{i}} r\left[\frac{a_{i+1}+a_{i}}{2}-\theta\right]^{2} d \theta \\
& =-w_{1}-\frac{r}{12 a_{1}} \sum_{i=1}^{2}\left(a_{i}-a_{i+1}\right)^{3} \\
& =-w_{1}-\frac{r}{12 a_{1}}\left(a_{2}^{3}+\left(a_{1}-a_{2}\right)^{3}\right) .
\end{aligned}
$$

Clearly, $u^{*}\left(w_{1}\right)$ takes the maximum value $u^{*}$ at $w_{1}=w$.

On the other hand, by paying $w_{1}<w$ at stage 2 in period 1 where $m_{1}<a_{1}$, she obtains $\bar{u}\left(w_{1}\right)$ :

$$
\begin{aligned}
\bar{u}\left(w_{1}\right) & =-w_{1}+\int_{0}^{a_{1}} \frac{1}{a_{1}} U^{R}\left(\frac{a_{1}}{2}, \theta\right) d \theta \\
& =-w_{1}-\frac{r}{a_{1}} \int_{0}^{a_{1}}\left(\frac{a_{1}}{2}-\theta\right)^{2} d \theta \\
& =-w_{1}-\frac{a_{1}^{2}}{12} r .
\end{aligned}
$$

Clearly, $\bar{u}\left(w_{1}\right)$ takes the maximum value $\bar{u}$ at $w_{1}=0$. Therefore, paying $w$ is an optimal decision for $R$ if and only if $u^{*} \geq \bar{u}$. Using condition (9), we have

$$
u^{*} \geq \bar{u} \Longleftrightarrow r\left(\frac{\left\{a_{1}(w)\right\}^{2}}{16}-b^{2}\right) \geq w .
$$

Since $a_{1}(w) \equiv \frac{2}{3}\left\{2-\sqrt{(1+6 b)^{2}-12 w / s}\right\}$, for any $w \in(w(4 b), w(1))$

- $a_{1}(w)$ is strictly increasing in $w$;

- $\frac{\left\{a_{1}(w)\right\}^{2}}{16}-b^{2}>0$ and $\frac{\left\{a_{1}(w(4 b))\right\}^{2}}{16}-b^{2}=0$;

- $\frac{d^{2}}{d w^{2}}\left\{a_{1}(w)\right\}^{2}>0$. 
Hence, if $r\left(\left\{a_{1}(w(1))\right\}^{2} / 16-b^{2}\right)>w(1)$, then there exists $\underline{w} \in(w(4 b), w(1))$ such that for all $w \in[\underline{w}, w(1))$, the inequality (10) holds.

Since $a_{1}(w(1))=1$ and $w(1)=s(1+12 b)(3+12 b) / 48$, the inequality $r\left(\left\{a_{1}(w(1))\right\}^{2} / 16-\right.$ $\left.b^{2}\right)>w(1)$ can be simplified into

$$
\frac{s}{r}<\frac{1-4 b}{1+12 b}
$$

Summarizing the above, we can conclude that if $s / r<(1-4 b) /(1+12 b)$, then the strategy profile and the system of beliefs that we construct above constitute an equilibrium when $w \in[\underline{w}, w(1))$ and boundaries of partition satisfies the condition (9).

Remark 1. In any equilibrium where $R$ pays positive amount of money to $S$ on the equilibrium path, meaningful information transmission must occur after $R$ pays money to $S$. Therefore, there must exist a partially informative equilibrium after $R$ pays $w$ to $S$. If it is not so, messages sent by $S$ in period 2 are completely noninformative. Consequently, $R$ has no incentive to pay money to $S$ in period 1. For this reason, in Proposition 4 , we require that $b<1 / 4$.

We can consider a possibility of the existence of a 3-element partition equilibrium where $S$ reveals the interval in a different order. Specifically, consider the following strategy profile. In period $1, S$ conveys $\theta \geq a_{2}$ or not. If $S$ conveys $\theta \geq a_{2}$, then $R$ pays $\tilde{w}$, and then, $S$ conveys $\theta<a_{1}$ or not. Note that $a_{2}<a_{1}$. The following Proposition 5 shows that there is no equilibrium where information is transmitted in such a way.

Proposition 5. Fix $b \in(1 / 12,1 / 4)$. Then, there exists no 3-element partition equilibrium where information is transmitted in the following steps. First, $S$ conveys that $\theta$ belongs to which element of $\left\{\left[0, a_{2}\right),\left[a_{2}, 1\right]\right\}$. Second, $S$ conveys that $\theta$ belongs to which element of $\left\{\left[a_{2}, a_{1}\right),\left[a_{1}, 1\right]\right\}$.

Proof of Proposition 5. For $S^{\prime}$ s incentive compatibility in period 2, the partition $\left\{\left[a_{2}, a_{1}\right),\left[a_{1}, 1\right]\right\}$ must be coincide with the 2-element equilibrium partition achieved in a model with one shot information transmission where $\theta$ is drawn from the uniform distribution over $\left[0, a_{1}\right)$. By Crawford and Sobel, it must be satisfied that $1=2 a_{1}-a_{2}+4 b$. This equation can be simplified into

$$
1-a_{1}=a_{1}-a_{2}+4 b .
$$

Moreover, similar to the condition (5), the indifference condition for $S$ of type $\theta=a_{2}$ induces the following condition:

$$
\tilde{w}=s\left\{\left(a_{2}+a_{1}\right) / 2-\left(a_{2}+b\right)\right\}^{2}-s\left(a_{2} / 2-\left(a_{2}+b\right)\right)^{2} .
$$

The value of $\tilde{w}$ is positive if and only if $a_{1}-a_{2}>a_{2}+4 b$. This means that $a_{1}-a_{2}>4 b$. Hence, we have

$$
\begin{aligned}
\left(a_{2}-0\right)+\left(a_{1}-a_{2}\right)+\left(1-a_{1}\right) & =2\left(a_{1}-a_{2}\right)+4 b+a_{2} \\
& >12 b+3 a_{2} .
\end{aligned}
$$


Since we now suppose that $b \in(1 / 12,1 / 4)$, we have $12 b+3 a_{2}>1$. Therefore, boundaries of the partition and the payment $\tilde{w}$ is not well defined. This implies that we cannot construct a 3-element partition equilibrium under which $R$ learns $\theta<a_{2}$ or not in period 1, and after learning $\theta \geq a_{2}$ in period 1, then $R$ learns in period 2 whether $\theta$ belongs to $\left[a_{2}, a_{1}\right)$ or $\left[a_{1}, 1\right]$.

It can be confirmed that if $r$ is large relative to $s, R$ can obtain greater ex ante expected revenue from the project in a 3-element partition equilibrium than that in 2-element partition equilibrium where the communication is one-shot. For details, see the proof of the following Lemma 1 . This implies that $R$ can obtain more valuable information about $S$ 's type through multistage information transmission with voluntary monetary transfer. However, it is not true that multistage information transmission with voluntary monetary transfer is always beneficial to $R$ since she has to pay money to $S$ in order to obtain valuable information.

We now show the second result that when $r$ is large relative to $s$, multistage information transmission with voluntary monetary transfer is more beneficial to both $R$ and $S$ than one-shot information transmission.

Proposition 6. Fix $b \in(1 / 12,1 / 4)$. Then there exists a positive value $\eta^{*}(b)$ such that if $\frac{s}{r}<$ $\eta^{*}(b)$, there exists a 3-element partition equilibrium whose outcome ex ante Pareto-dominates all the equilibrium outcomes in the model with one-shot information transmission.

Suppose that $s / r<(1-4 b) /(1+12 b)$. Fix a 3-element partition equilibrium constructed in the proof of Proposition 4. We prove Proposition 6 by two steps. First, we show that if $r$ is large relative to $s$, there exists a 3-element partition equilibrium which $R$ prefers to all the equilibria without monetary transfer.

CS have shown that for given $b$, in a model with one-shot information transmission, $R$ always strictly prefers 2-element partition equilibrium to the babbling equilibrium. Let $\left\{\left[\tilde{a}_{2}, \tilde{a}_{1}\right)\left[\tilde{a}_{1}, \tilde{a}_{0}\right]\right.$ be the partition of 2-element partition equilibrium in a model with one-shot information transmission. We denote by $E \hat{U}^{R}$ the ex ante expected payoff of $R$ under this equilibrium. We have

$$
\begin{aligned}
E \hat{U}^{R} & =-\sum_{i=1}^{2} \int_{\tilde{a}_{i}}^{\tilde{a}_{i-1}} r\left[\frac{\tilde{a}_{i-1}+\tilde{a}_{i}}{2}-\theta\right]^{2} d \theta \\
& =-\frac{r}{48}-r b^{2} .
\end{aligned}
$$

We denote by $E \bar{U}^{R}\left(a_{1}\right)$ the ex ante expected payoff of $R$ under the 3-element partition equilibrium with $a_{1} \in(\underline{a}, 1)$ where $\underline{a} \equiv a_{1}(\underline{w})$.

By the definition of $a_{1}(w)$, we have

$$
w(x) \equiv a_{1}^{-1}(x)=s[3(1 / 2+b-x / 4)(x / 4+b-3 / 2)] . \text { for } x \in[\underline{a}, 1) .
$$

In what follows, we denote by $s \cdot \alpha(b, x)$ the function $w(x)$. 
The ex ante expected payoff of $R$ under 3-element partition equilibrium where $a_{1}=x \in(\underline{a}, 1)$ is

$$
\begin{aligned}
E \bar{U}^{R}(x) & =-\sum_{i=1}^{3} \int_{a_{i}(x)}^{a_{i-1}(x)} r\left[\frac{a_{i-1}(x)+a_{i}(x)}{2}-\theta\right]^{2} d \theta-x_{S} \cdot \alpha(b, x) \\
& =-r\left\{\frac{x^{3}}{48}+x b^{2}\right\}-\frac{r}{12}(1-x)^{3}-x_{S} \cdot \alpha(b, x) .
\end{aligned}
$$

We show the following Lemma 1.

Lemma 1. There exists a positive value $\eta^{*}(b)$ such that if $s / r<\eta^{*}(b)$, there exists $\bar{x}, \underline{x} \in(\underline{a}, 1)$ such that

$$
E \bar{U}^{R}(\bar{x})>E \hat{U}^{R} \text { and } E \bar{U}^{R}(\underline{x})<E \hat{U}^{R} .
$$

Proof of Lemma 1. Let $\delta(x, s, r) \equiv \frac{1}{r}\left\{E \bar{U}^{R}(x)-E \hat{U}^{R}\right\}$. We have

$$
\delta(x, s, r)=-\frac{1}{16}\left(1-x^{3}\right)+\frac{x}{4}(1-x)+b^{2}(1-x)-\frac{s}{r} x \alpha(b, x) .
$$

It holds that $\delta(x, s, r)<0$, if and only if

$$
\eta^{*}(b, x) \equiv \frac{-\frac{1}{16}\left(1-x^{3}\right)+\frac{x}{4}(1-x)+b^{2}(1-x)}{x \alpha(b, x)}<\frac{s}{r} .
$$

Since $\left.\frac{\partial}{\partial x}\right|_{x=1} \eta^{*}(b, x)<0$ and $\eta^{*}(b, 1)=0$, there exists $\hat{\varepsilon}>0$ such that $\eta^{*}(b, \underline{x})<s / r$ for all $\underline{x} \in(1-\hat{\varepsilon}, 1)$.

It holds that $\delta(x, s, r)>0$, if and only if

$$
\eta^{*}(b, x)>\frac{s}{r} \text {. }
$$

Since $\inf _{x \in(a, 1)} x \alpha(b, x)>0, \eta^{*}(b, x)$ has a least upper bound $\eta^{*}(b)=\sup _{x \in(a, 1)} \eta^{*}(b, x)>0$. Therefore, if $s / r<\eta^{*}(b)$, there exists $\bar{x}$ such that $\bar{x} \in(\underline{a}, 1)$ and $\delta(\bar{x})>0$. This completes the proof of Lemma 1.

Remark 2. When $x$ almost equal to 1, boundaries of the 3-element partition equilibrium almost coincides with boundaries of the 2-element partition equilibrium in a model with one-shot information transmission. Nevertheless, the payment of monetary transfer $a_{1}^{-1}(x)$ is high (almost coincides with $\left.w(1)\right)$. Therefore, if $s / r<(1-4 b) /(1+12 b)$, there always exists a 3-element partition equilibrium which is unfavorable to $R$. Moreover, since $\underline{w}$ goes to $w(1)$ as $s / r$ goes to $(1-4 b) /(1+12 b), \underline{a}$ goes to 1 as $s / r$ goes to $(1-4 b) /(1+$ $12 b)$. Hence, $\eta^{*}(b)$ is always less than $(1-4 b) /(1+12 b)$.

Hereafter, we complete the proof of Proposition 6. CS have shown that for given $b$, in a model with one-shot information transmission, $S$ always strictly prefers ex ante 2-element partition equilibrium to the babbling equilibrium. We denote by $E \hat{U}^{S}$ the ex ante expected payoff of $S$ under the 2-element partition equilibrium in a model with one-shot information transmission. We have

$$
\begin{aligned}
E \hat{U}^{S} & =-\sum_{i=1}^{2} \int_{\tilde{a}_{i}}^{\tilde{a}_{i-1}} s\left[\frac{\tilde{a}_{i-1}+\tilde{a}_{i}}{2}-\theta\right]^{2} d \theta-s b^{2} \\
& =\frac{s}{r} E \hat{U}^{R}-s b^{2} .
\end{aligned}
$$


We denote by $\bar{E} U^{S}(x)$ the ex ante expected payoff of $S$ under the 3-element partition equilibrium with $a_{1}=x \in(\underline{a}, 1)$.

$$
\begin{aligned}
E \bar{U}^{S}(x) & =-\sum_{i=1}^{3} \int_{a_{i}(x)}^{a_{i-1}(x)} s\left[\frac{a_{i-1}(x)+a_{i}(x)}{2}-\theta\right]^{2} d \theta-s b^{2}+x \cdot w(x) \\
& =\frac{s}{r}\left\{E \bar{U}^{R}(x)+x \cdot w(x)\right\}-s b^{2}+x \cdot w(x) .
\end{aligned}
$$

Clearly, if $E \bar{U}^{R}(x)>E \hat{U}^{R}$, then $E \bar{U}^{S}(x)>E \hat{U}^{S}$. Therefore, if $s / r<\eta^{*}(b)$, there exists $x \in(\underline{a}, 1)$ such that

$$
E \bar{U}^{R}(x)>E \hat{U}^{R} \text { and } E \bar{U}^{S}(x)>E \hat{U}^{S} .
$$

This completes the proof of Proposition 6.

It is well known that the existence of non-strategic mediator leads to improved information transmission. Now, we compare our communication procedure with optimal mediation. In mediation model $S$ can send a message to an impartial mediator, who then passes on a recommendation to $R$ according to some predetermined rule. Goltsman et al. (2009) have characterized the optimal mediation where $R$ 's ex ante expected payoff is $-\frac{r}{3} b(1-b)$. The following Proposition 7 shows that under 2-period information elicitation with voluntary monetary transfer, $R$ can obtain higher ex ante expected payoff than that under optimal mediation model.

Proposition 7. Fix $b \in(1 / 12,\{4+\sqrt{3}\} / 26) .{ }^{15}$ Then there exists $\eta^{\prime}(b)$ such that if $s / r<\eta^{\prime}(b)$, for some $x \in(\underline{a}, 1)$,

$$
E \bar{U}^{R}(x)>-\frac{r}{3} b(1-b) .
$$

Since this proposition can be proved in the same way as the proof of Lemma 1, the formal proof is omitted. When $b$ is almost equal to $1 / 4$, boundaries of the 3 element partition equilibrium almost coincides with those of the 2-element partition equilibrium in the CS model. The value of $-\frac{r}{3} b(1-b)$ is always strictly higher than the $R$ 's equilibrium payoff under the 2-element partition equilibrium in the CS model. Therefore, Proposition 7 requires that $b<(4+\sqrt{3}) / 26<1 / 4$.

\subsection{Effective T-period Communication}

Under the equilibrium that we construct in previous Section 2.5.1, information is transmitted within only two periods, the first period and the second period, regardless of the length of communication. It seems that $R$ does not use $T$-period communication effectively. In Section 2.5.2, we show the benefit of long term communication.

First, we provide some properties of equilibrium outcomes. The following Proposition 8 shows that all the equilibria in $\Gamma(b, s, r, T)$ are finite partition equilibria.

${ }^{15}$ Note that $\frac{1}{5}<\frac{1}{26}(4+\sqrt{3})<\frac{1}{4}$. 
Proposition 8. In the uniform-quadratic case, all the equilibria in $\Gamma(b, s, r, T)$ are finite partition equilibria.

The proof of Proposition 8 can be found in Appendix C. Second, we provide an upper bound of $R^{\prime}$ s equilibrium payoff. The following Proposition 9 establishes an upper bound ${ }^{16}$ of $R^{\prime}$ s equilibrium payoff.

Proposition 9. In the uniform-quadratic case, the upper bound of $R^{\prime}$ 's equilibrium payoff is given by $-16 r b^{3} / 3$.

The proof of Proposition 9 can be found in Appendix D. One of the main findings in our analysis is that under some conditions, this upper bound $-16 r b^{3} / 3$ can be approximated by $R^{\prime}$ s payoff under a finite partition equilibrium when $T$ is long enough. For the details of this result, see Proposition 11.

Next, we demonstrate that under a certain condition, there exists an equilibrium under which information is transmitted within the whole $T$ periods. More precisely, we consider the following information elicitation. In period $1, R$ learns whether the value of $\theta$ is less than $a_{1}$. If $R$ learns that the value of $\theta$ is less than $a_{1}$, then she pays a certain amount of money to $S$. After that, in period 2, $R$ learns whether the value of $\theta$ is less than $a_{2}$. If $R$ learns that the value of $\theta$ is less than $a_{2}$, then she pays a certain amount of money to $S$. This information elicitation is repeated until the last period in the communication round. In the last period in the communication round, $R$ learns whether the value of $\theta$ is less than $a_{T}$. Under this communication process, $R$ eventually learns to which element of a partition $\left\{\left[a_{i+1}, a_{i}\right)\right\}_{i=1}^{T} \cup\left[a_{1}, a_{0}\right]$ the state $\theta$ belongs. In what follows, we call this communication process (monotone) effective T-period communication. ${ }^{17}$

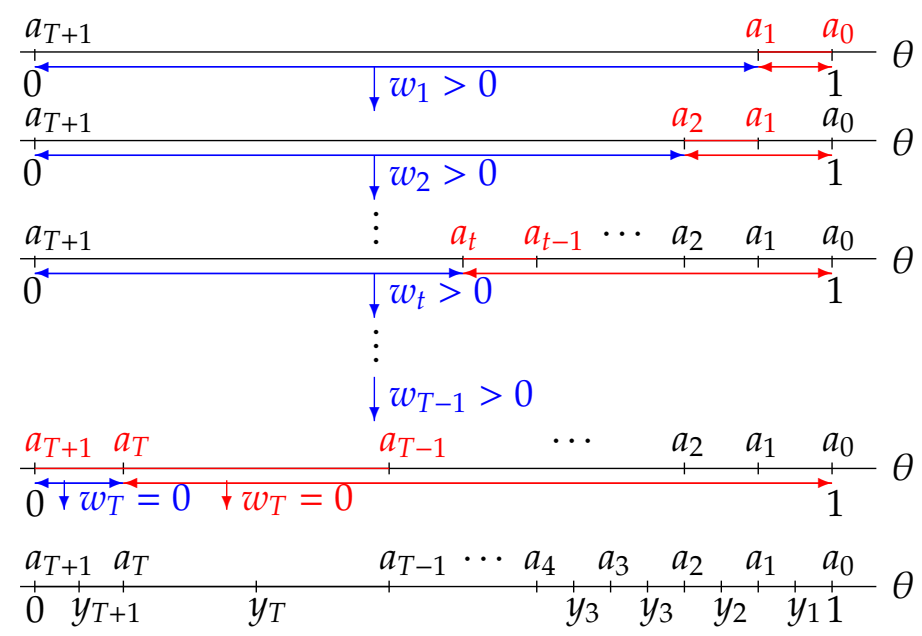

Figure 3: Information elicitation under monotone effective $T$-period communication

Proposition 10. Fix $b \in(0,1 / 4)$. If $s / r<(1-4 b) /(1+12 b)$, there exists an equilibrium with effective T-period communication.

\footnotetext{
${ }^{16}$ This upper bound is not necessarily the least upper bound.

${ }^{17}$ This Information elicitation is similar to those in Ivanov (2015) and Hörner and Skrzypacz (2016)
} 
To prove Proposition 10, we construct a $(T+1)$-element partition equilibrium with effective $T$-period communication. Under this equilibrium, the information is transmitted in the following steps. At $h^{(t, 1)}$ in which $m_{t^{\prime}}<a_{t^{\prime}}$ and $w_{t^{\prime}} \geq w_{t^{\prime}}^{*}$ for all $t^{\prime}<t, S$ of type $\theta<a_{t}$ sends a message $m_{t}$ randomly according to a uniform distribution over $\left[0, a_{t}\right)$, and $S$ of type $\theta \geq a_{t}$ sends a message $m_{t}$ randomly according to a uniform distribution over $\left[a_{t}, 1\right]$. Otherwise, any type of $S$ sends a message $m_{t}$ randomly according to a uniform distribution over $[0,1]$. If $R$ learns $\theta<a_{t}$ at stage 1 in period $\mathrm{t}$, then he pays $w_{t}^{*}$ to $S$. Otherwise, he pays nothing to $S$ at stage 2 .

Under the above communication procedure, for any $h^{T+1}$, the closure of $\{\theta \in \Theta$ : $\left.f\left(\theta \mid h^{T+1}\right)>0\right\}, I\left(h^{T+1}\right)$, belongs to $\left\{\left[a_{i+1}, a_{i}\right]\right\}_{i=0}^{T} \cup\left\{\left[a_{T+1}, a_{i}\right]\right\}_{i=0}^{T-1}$, and $R$ believes that $\theta$ is uniformly distributed over $I\left(h^{T+1}\right)$. Therefore, $R$ must choose $y=\frac{\min I\left(h^{T+1}\right)+\max I\left(h^{T+1}\right)}{2}$ at $h^{T+1}$.

At $h^{T}$ in which $m_{t^{\prime}}<a_{t^{\prime}}$ and $w_{t^{\prime}} \geq w_{t^{\prime}}^{*}$ for all $t^{\prime}<T$, since $R$ does not obtain additional information in the future, $w_{T}^{*}$ must be equal to 0 . Therefore, $\left\{\left[a_{i+1}, a_{i}\right)\right\}_{i=T-1}^{T}$ must coincide with the 2-element equilibrium partition achieved in a model with one shot information transmission where $\Theta=\left[0, a_{T-1}\right)$. By Crawford and Sobel, it must be satisfied that

$$
a_{T}=\frac{a_{T-1}}{2}-2 b \text {. }
$$

This implies that $a_{T-1}>4 b$. We define $a_{t}$ and $w_{t}^{*}$ as follows:

$$
\begin{aligned}
& a_{i} \equiv\left\{\begin{array}{l}
1-i a \text { for } i \in\{0, \ldots, T-1\}, \\
\frac{1-(T-1) a}{2}-2 b \text { for } i=T, \\
0 \quad \text { for } i=T+1 .
\end{array}\right. \\
& w_{t}^{*} \equiv\left\{\begin{array}{l}
2 b s a \text { for } t \in\{0, \ldots, T-2\}, \\
\frac{s}{16}\{1+12 b-a(T+1)\}\{1+4 b-a(T-3)\} \quad \text { for } t=T-1, \\
0 \text { for } t=T .
\end{array}\right.
\end{aligned}
$$

Note that $0=a_{T+1}<a_{T}<a_{T-1}<\cdots<a_{0}=1$ and $4 b<a_{T-1}$ if and only if $a<(1-4 b) /(T-1)$, and $w_{T-1}^{*}>0$ if $a<\min \{(1+12 b) /(T+1),(1+4 b) /(T-3)\}$. Since we suppose that $T \geq 3$, we have $(1+4 b) /(T-3)>(1-4 b) /(T-1)$. Therefore, if $a<\min \{(1-4 b) /(T-1),(1+12 b) /(T+1)\}, a_{i}$ and $w_{t}^{*}$ are well-defined. Moreover, for any $t \in\{1, \ldots, T-1\}, w_{t}^{*}$ becomes a solution to an equation,

$$
s\left(\frac{a_{t}+a_{t-1}}{2}-\left(a_{t}+b\right)\right)^{2}=-s\left(\frac{a_{t+1}+a_{t}}{2}-\left(a_{t}+b\right)\right)^{2}+w_{t}^{*}
$$

induced by a condition which requires that $S$ 's types who fall on the boundaries between adjacent intervals are indifferent between the associated values of $y$.

The strategy profile and system of beliefs outlined above, which we denote by $\xi_{T}$, can not always be an equilibrium. It depends on the value of $a$. We show that $\xi_{T}$ can be an equilibrium by taking $a$ small enough. When $a \approx 0, R^{\prime}$ s payment $w^{t}$ in each $t \leq T-2$ goes 0 . Consider a history at stage 2 in period $T-1$ where $m_{t}<a_{t}$ for all $t \leq T-1$. At such a history, there are two cheap talk equilibria in the remaining 
game; the babbling equilibrium and the 2-element partition equilibrium. Since we now suppose that $a \approx 0$, if the 2-element partition equilibrium is chosen in period $T$, $R$ 's continuation payoff is approximated by $-r\left(b^{2}-1 / 48\right)$. Otherwise, $R^{\prime}$ s continuation payoff is approximated by $-r / 12$. Moreover, $w_{T-1}^{*} \approx s(1+12 b)(1+4 b) / 16$. Since we suppose that $s / r<(1-4 b) /(1+12 b)$, we have

$$
-r\left(b^{2}-\frac{1}{48}\right)-\left(-\frac{r}{12}\right)>\frac{s}{16}(1+12 b)(1+4 b) .
$$

Thus, $R$ has incentive to pay $w_{T-1}^{*}$ at this history so that the babbling equilibrium would not be chosen in next period. The formal proof can be found in Appendix E.

In Proposition 10, we have only shown the possibility of the effective T-period communication. In order for $\xi_{T}$ to be an equilibrium, it might be required that $a_{T-1}$ is close to 1 . If $a_{T-1}$ is close to $1, R$ faces with the history $h^{(T, 1)}$ in which $I\left(h^{(T, 1)}\right)=\left[0, a_{T-1}\right]$ with a high probability on the equilibrium path. Moreover, $\left\{\left[a_{i+1}, a_{i}\right)_{i=T-1}^{T}\right\}$ almost coincides with the 2-element equilibrium partition achieved in a model with one-shot information transmission. In such a case, the initial $(T-1)$-period communication does not have much meaning for $R$ ex ante. Now, we show that the benefit of long therm communication.

Proposition 11. Fix $b \in(0,1 / 4)$ and $r>0$. For any $d>0$, there exists $\bar{T}(b, d, r)$ such that if $T \geq \bar{T}(b, d, r)$, there exists $\underline{\eta}(b, T, d, r)$ such that if $s<\underline{\eta}(b, T, d, r), R$ can obtain the higher ex ante expected payoff than $-\overline{1} 6 r b^{3} / 3-d$.

The formal proof can be found in Appendix F. We have already shown that an upper bound of $R^{\prime}$ s equilibrium payoff is $-16 r b^{3} / 3$. This Proposition 11 shows that if the length of the communication round is sufficiently high and $R$ places greater importance on the project than $S$ does, then this upper bound can be approximated by an $R^{\prime}$ s equilibrium payoff.

Remark 3. Suppose that $\xi_{\varepsilon}$ constitutes an equilibrium of $\Gamma(b, s, r, T)$ where $R$ obtains $E U^{R}(\varepsilon)>-16 r b^{3} / 3-d$. Consider $\Gamma\left(b, s, r, T^{\prime}\right)$ where $T^{\prime}>T$. Now, construct a strategy profile $\xi_{\varepsilon}^{\prime}$ by modifying $\xi_{\varepsilon}$. In particular, under $\xi_{\varepsilon}^{\prime}$, players follow $\xi_{\varepsilon}$ until period $\mathrm{T}$, and then $S$ always sends babbling massage and $R$ never pays money to $S$ in the future. It is obvious that $\xi_{\varepsilon}^{\prime}$ constitutes an equilibrium of $\Gamma\left(b, s, r, T^{\prime}\right)$ and $R^{\prime}$ s equilibrium payoff is equal to $E U^{R}(\varepsilon)>-16 r b^{3} / 3-d$.

\subsection{Comparison with Other Communication Protocols}

Now, we compare the effective $T$-period communication with other communication protocols, i.e. delegation, arbitration and mediation, that strictly dominate the oneshot information transmission: that is, under these communication protocols, $R$ can obtain the higher ex ante expected payoff than that under the one-shot information transmission. 
When $R$ delegates control, her payoff is given by $-r b^{2}$. As shown by $C S$, the ex ante expected payoff of $R$ under the one shot information transmission is given by

$$
E U_{C S}^{R}=-r\left(\frac{1}{12 n^{2}}+\frac{b^{2}\left(n^{2}-1\right)}{3}\right) .
$$

The maximum number of partition equilibrium outcomes $\tilde{n}$ is given by

$$
\tilde{n} \equiv\left\lceil-\frac{1}{2}+\frac{1}{2}\left(1+\frac{2}{b}\right)^{\frac{1}{2}}\right\rceil,
$$

where $\lceil x\rceil$ denotes the smallest integer greater than or equal to $x$. We can immediately verify that $E U_{C S}^{R}<-r b^{2}$ for $n \geq 2$, and thus $R$ prefers delegation to the one shot information transmission whenever informative communication is possible.

In contrast, under effective $T$-period communication, $R$ can obtain the ex ante expected payoff as well as $-16 r b^{3} / 3$ when $T$ is sufficiently large and $s$ is small relative to $r$. We can verify immediately that $-16 r b^{3} / 3>-r b^{2}$ when $b<3 / 16$.

Next, consider a communication protocol arbitration where the players can send messages to a neutral third party (arbitrator), and after having received the messages, the arbitrator announces an project. This announcement serves as a binding recommendation to $R$. Namely, $R$ cannot choose any action that is different from the recommended one. Goltsman et al. (2009) characterizes the optimal arbitration rule and shows that the $R^{\prime}$ s ex ante expected payoff under optimal arbitration is $-r b^{2}(1-4 b / 3) .{ }^{18}$ We can immediately verify that $-16 r b^{3} / 3>-r b^{2}(1-4 b / 3)$ when $b<3 / 20$.

Therefore, Proposition 11 implies that when the length of the communication round is high and $R$ places greater importance on the project than $S$ does, $R$ can obtain higher ex ante expected payoff under effective $T$-period communication than those under delegation and arbitration. ${ }^{19}$

\section{Generalization of Proposition 4 and Proposition 6}

In this section, we show the following two results under the more general settings where the players' payoff functions and the prior probability of the state are not restricted to the uniform-quadratic case but those described in Section 2. One is that if $T \geq 2$ and $r$ is large relative to $s, R$ can obtain more detailed information in our model than that in a model with one-shot information transmission. The other is that if $T \geq 2$ and $r$ is large relative to $s$, there exists an equilibrium whose outcome ex ante Pareto-dominates all equilibrium outcomes in a model with one-shot information transmission.

We now show that the first result. Recall that $\tilde{n}(b)$ denotes the maximum number of elements of equilibrium partition achievable in a model with one-shot information transmission. As can be seen from the uniform-quadratic case, under our equilibrium construction in Proposition 4, it is necessary that after $S$ conveys some information in

\footnotetext{
${ }^{18}$ Having restricted attention to deterministic mechanism, Melumad and Shibano (1991) provided the optimal arbitration (optimal delegation) rule.

${ }^{19}$ Since the optimal arbitration rule dominates the optimal mediation rule, our communication protocol could also strictly dominate the optimal mediation rule.
} 
period 1, there are multiple equilibria in the remaining game. Therefore, we assume that $\tilde{n}(b) \geq 2$. In a model with one-shot information transmission, if Condition $\mathrm{M}$ holds, then the most informative equilibrium is $\tilde{n}(b)$-element partition equilibrium where $\left\{\left[\tilde{a}_{\tilde{n}(b)}, \tilde{a}_{\tilde{n}(b)-1}\right), \ldots\left[\tilde{a}_{1}, \tilde{a}_{0}\right]\right\}$, and $0=\tilde{a}_{\tilde{n}(b)}<\tilde{a}_{\tilde{n}(b)-1}<\cdots<\tilde{a}_{1}<\tilde{a}_{0}=1$.

The following Proposition 12 establishes that in multistage information transmission with voluntary monetary transfer, there exists an equilibrium whose partition has more steps that that in a model with one-shot information transmission.

Proposition 12. Fix $b>0$ and suppose that $\tilde{n} \equiv \tilde{n}(b) \geq 2$. Then, there exists a positive value $\eta(b)$ such that if $s / r<\eta(b)$, then there exists an equilibrium with a $(\tilde{n}+1)$-element partition.

To prove this Proposition, we construct a strategy profile that induces a $(\tilde{n}+1)$ element partition: $\left\{\left[\hat{a}_{\tilde{n}+1}, \hat{a}_{\tilde{n}}\right), \ldots\left[\hat{a}_{1}, \hat{a}_{0}\right]\right\}$, and $0=\hat{a}_{\tilde{n}+1}<\hat{a}_{\tilde{n}}<\cdots<\hat{a}_{1}<\hat{a}_{0}=1$. The following strategy profile is an extension of the strategy profile that we construct in Section 5.1.

At stage 1 in period $1, S$ of type $\theta \geq \hat{a}_{1}$ sends a message $m_{1}$ randomly according to a uniform distribution over $\left[\hat{a}_{1}, 1\right]$, and $S$ of type $\theta<\hat{a}_{1}$ sends a message $m_{1}$ randomly according to a uniform distribution over $\left[0, \hat{a}_{1}\right)$. If $m_{1}<\hat{a}_{1}$, then $R$ pays a certain amount of money, $w^{*}$, to $S$ at stage 2 in period 1 . Otherwise, she pays nothing to $S$. If $m_{1}<\hat{a}_{1}$ and $w_{1} \geq w^{*}$, then, at stage 1 in period $2, S$ of type $\theta \geq \hat{a}_{2}$ sends a message $m_{2}$ randomly according to a uniform distribution over $\left[\hat{a}_{2}, 1\right]$, and $S$ of type $\theta \in\left[\hat{a}_{i+1}, \hat{a}_{i}\right)$, for $i \in\{2, \ldots, \tilde{n}\}$, sends a message $m_{2}$ randomly according to a uniform distribution over $\left[\hat{a}_{i+1}, \hat{a}_{i}\right)$. Otherwise, $S$ sends a message $m_{2}$ randomly according to uniform distribution over $[0,1]$ regardless of his type. In period $t \geq 2, R$ always pays nothing to $S$. In period $t \geq 3, S$ always sends babbling message. In period $T+1, R$ chooses a project $\rho\left(h^{T+1}\right) \equiv \arg \max _{y} \int u^{R}(y, \theta) f\left(\theta \mid h^{T+1}\right) d \theta$. In what follows, we denote by $(\hat{\sigma}, \hat{\rho})$ the strategy profile defined above, and denote by $\hat{f}$ the belief system derived from $(\hat{\sigma}, \hat{\rho})$.

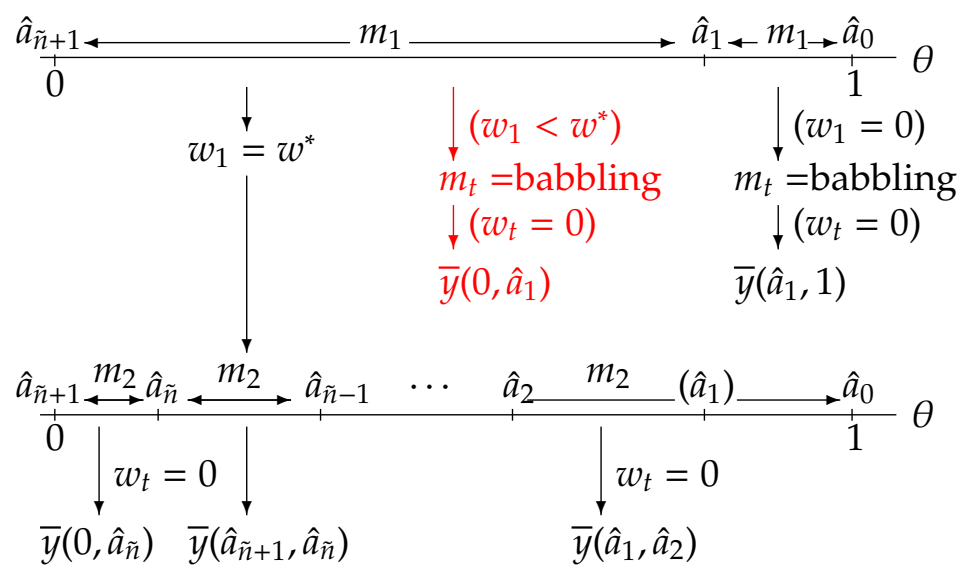

Figure 4: Equilibrium 
Under the above strategy profile, we have to take an equilibrium partition whose boundaries $\left\{\hat{a}_{\tilde{n}}, \ldots, \hat{a}_{2}\right\}$ coincide with those of the $\tilde{n}$-element partition equilibrium in the CS model where state space is $\left[0, \tilde{a}_{1}\right)$. Moreover, it must be satisfied that $w^{*}=$ $s \cdot u^{S}\left(\bar{y}\left(a_{2}, a_{1}\right), a_{1}, b\right)-s \cdot u^{S}\left(\bar{y}\left(a_{1}, 1\right), a_{1}, b\right)>0$. Therefore, if $\theta$ is close to $\hat{a}_{1}$ and $\theta<\hat{a}_{1}$, then $S$ strictly prefers the project $\bar{y}\left(\hat{a}_{1}, 1\right)$ to the project $\bar{y}\left(\hat{a}_{2}, \hat{a}_{1}\right)$. Hence, $R$ pays money $w^{*}$ after receiving the message that " $m_{1}<\hat{a}_{1}$ " so that $S$ 's type $\theta<\hat{a}_{1}$ would not lie to her. Since $R$ cannot commit to compensate for $S$ 's message, the following inequality must be hold for paying $w^{*}$ to be optimal for $R$.

$$
\frac{r}{G\left(\hat{a}_{1}\right)} \sum_{i=1}^{\tilde{n}} \int_{\hat{a}_{i+1}}^{\hat{a}_{i}} u^{R}\left(\bar{y}\left(\hat{a}_{i+1}, \hat{a}_{i}\right), \theta\right) g(\theta) d \theta-\frac{r}{G\left(\hat{a}_{1}\right)} \int_{0}^{\hat{a}_{1}} u^{R}\left(\bar{y}\left(0, \hat{a}_{1}\right), \theta\right) g(\theta) d \theta \geq w^{*} .
$$

The left-hand side of this inequality represents the value of additional information, that is, the value of the partition $\left\{\left[0, \hat{a}_{\tilde{n}}\right), \ldots,\left[\hat{a}_{2}, \hat{a}_{1}\right)\right\}$, that $R$ receives in period 2 by paying $w^{*}$ after receiving $m_{1}<\hat{a}_{1}$. It is obvious that $R$ always strictly prefers the partition $\left\{\left[0, \hat{a}_{\tilde{n}}\right), \ldots,\left[\hat{a}_{2}, \hat{a}_{1}\right)\right\}$ to the partition $\left\{\left[0, \hat{a}_{1}\right)\right\}$. This implies that the left-hand side of the inequality (12) is positive and increasing in $r$ when $\tilde{n} \geq 2$. Moreover, since $w^{*}=s \cdot u^{S}\left(\bar{y}\left(a_{1}, 1\right), a_{1}, b\right)-s \cdot u^{S}\left(\bar{y}\left(a_{2}, a_{1}\right), a_{1}, b\right),{ }^{20}$ the right-hand side of the inequality (12) is decreasing and goes to 0 as $s$ goes to 0 . Therefore, if $r$ is large enough relative to $s$, then $R$ has incentive to pay $w^{*}$ after receiving the message $m_{1}<\hat{a}_{1}$.

In Appendix $\mathrm{G}$, we make sure that there exists $\eta(b)>0$ such that if $\frac{s}{r}<\eta(b)$, by taking the boundaries of the partition $\left\{\left[\hat{a}_{\tilde{n}+1}, \hat{a}_{\tilde{n}}\right), \ldots\left[\hat{a}_{1}, \hat{a}_{0}\right]\right\}$ suitably, $((\hat{\sigma}, \hat{\rho}), \hat{f})$ constitutes an equilibrium.

Next, we show that under some conditions, multistage information transmission with voluntary monetary transfer is more beneficial to both $R$ and $S$ than one-shot information transmission. To see this, we focus on the equilibrium, $((\hat{\sigma}, \hat{\rho}), \hat{f})$, that we constructed to prove the Proposition 12.

Let $\left\{\left[\hat{a}_{\tilde{n}+1}^{x}, \hat{a}_{\tilde{n}}^{x}\right), \ldots\left[\hat{a}_{1}^{x}, \hat{a}_{0}^{x}\right]\right\}$ be the partition whose boundaries $\left\{\hat{a}_{\tilde{n}^{\prime}}^{x} \ldots, \hat{a}_{2}^{x}\right\}$ coincide with those of the $\tilde{n}$-element partition equilibrium in the CS model where state space is $[0, x)$.

We denote by $E \hat{U}^{R}(x)$ the ex ante expected payoff of $R$ under $((\hat{\sigma}, \hat{\rho}), \hat{f})$ with $(\tilde{n}+1)$ element partition: $\left\{\left[\hat{a}_{\tilde{n}+1}^{x}, \hat{a}_{\tilde{n}}^{x}\right), \ldots\left[\hat{a}_{1}^{x}, \hat{a}_{0}^{x}\right]\right\}$ where $\hat{a}_{1}^{x} \equiv x \in\left(\underline{a}_{1}(s / r), 1\right)$. Let $\underline{a}_{1}(s / r)$ be the infimum value of $z$ such that (12) holds for all $x \in(z, 1){ }^{21}$

We have

$$
E \hat{U}^{R}(x)=\hat{W}(x)-E\left[w^{*}\right] .
$$

where $\hat{W}(x)$ denotes $R$ 's ex ante expected utility from project:

$$
\hat{W}(x) \equiv r \sum_{i=1}^{\tilde{n}+1} \int_{\hat{a}_{i}^{x}}^{\hat{a}_{i-1}^{x}} u^{R}\left(\bar{y}\left(\hat{a}_{i}^{x}, \hat{a}_{i-1}^{x}\right), \theta\right) g(\theta) d \theta .
$$

CS have shown that in a model with one-shot information transmission, under Condition $\mathrm{M}, R$ always strictly prefers $\tilde{n}$-element partition equilibrium to any other equilibria.

\footnotetext{
${ }^{20}$ Recall the Figure 2 in Section 5.1.

${ }^{21}$ The formal characterization of $\underline{a}_{1}(s / r)$ can be found in Appendix G.
} 
We denote by $E U_{C S}^{R}$ the ex ante expected payoff of $R$ under the $\tilde{n}$-element partition equilibrium in a model with one-shot information transmission. We have

$$
E U_{C S}^{R}=r \sum_{i=1}^{\tilde{n}} \int_{\tilde{a}_{i}}^{\tilde{a}_{i-1}} u^{R}\left(\bar{y}\left(\tilde{a}_{i}, \tilde{a}_{i-1}\right), \theta\right) g(\theta) d \theta .
$$

The boundaries $\left\{\hat{a}_{\tilde{n}+1}^{x}, \hat{a}_{\tilde{n}}^{x}, \ldots, \hat{a}_{1}^{x}\right\}$ almost coincides with the boundaries $\left\{\tilde{a}_{\tilde{n}}, \tilde{a}_{\tilde{n}-1}, \ldots, \tilde{a}_{0}\right\}$ induced by $\tilde{n}$-element partition equilibrium in a model with one-shot information transmission when $\hat{a}_{1}^{x} \approx 1$. Therefore, we have $\lim _{x \uparrow 1} \hat{W}(x)=E U_{C S}^{R}$. This implies that $\hat{W}(x)>E U_{C S}^{R}$ for some $x \in\left(\underline{a}_{1}(s / r), 1\right)$ when the following Condition $C$ holds.

Condition C. $\left.\frac{d}{d x}\right|_{x=1} \hat{W}(x)<0$.

In what follows, we restrict attention to $\left(\left(U^{R}, U^{S}\right), G\right)$ under which Condition $C$ holds. Note that there exists a pair of players payoff functions and the prior distribution of state, $\left(\left(U^{R}, U^{S}\right), G\right)$, under which Condition $C$ holds. For details, see Appendix K. It is not true that Condition M implies that Condition C. In Remark 8 in Appendix K, we provide an example in which Condition $\mathrm{M}$ is satisfied, while Condition $\mathrm{C}$ is not.

Under this condition, for some $\hat{a}_{1}^{x} \equiv x \in\left(\underline{a}_{1}(s / r), 1\right)$, the partition $\left\{\left[\hat{a}_{\tilde{n}+1}^{x}, \hat{a}_{\tilde{n}}^{x}\right), \ldots\left[\hat{a}_{1}^{x}, \hat{a}_{0}^{x}\right]\right\}$ is more informative for $R$ with respect to the project than the partition $\left\{\left[\tilde{a}_{\tilde{n}}, \tilde{a}_{\tilde{n}-1}\right), \ldots\left[\tilde{a}_{1}, \tilde{a}_{0}\right]\right\}$. However, even when Condition C holds, it is not true that multistage information transmission with voluntary monetary transfer is always beneficial to $R$ since she has to pay money $w^{*}$ to $S$ in order to obtain valuable information.

We now show the following Proposition 13.

Proposition 13. Fix $b>0$ and suppose that $\tilde{n} \geq 2$ and Condition $C$ holds. Then there exists $a$ positive value $\tilde{\eta}(b)$ such that if $s / r<\tilde{\eta}(b)$, there exists $a(\tilde{n}+1)$-element partition equilibrium whose outcome ex ante Pareto-dominates all the equilibrium outcomes in a model with one-shot information transmission.

We prove Proposition 13 by three steps. Let $((\hat{\sigma}, \hat{\rho}), \hat{f})$ be a partition equilibrium constructed in Proposition 12. First, we show that if $\frac{s}{r}<\eta(b)$, there exists a $(\tilde{n}+1)$ element partition equilibrium which $S$ prefers to all equilibria in $\Gamma(b, s, r, 1)$. This result can be summarized as Lemma 2. Second, we show that there exists a positive value $\bar{\eta}(b)$ such that if $\frac{s}{r}<\bar{\eta}(b)$, there exists a $(\tilde{n}+1)$-element partition equilibrium which $R$ prefers to all equilibria in $\Gamma(b, s, r, 1)$. This result can be summarized as Lemma 3. Finally, we show that there exists a positive value $\tilde{\eta}(b)$ such that if $\frac{s}{r}<\tilde{\eta}(b)$, the intersection of the following two sets is nonempty: the set of $(\tilde{n}+1)$-element partition equilibria which $S$ prefers to all equilibria in $\Gamma(b, s, r, 1)$ and the set of $(\tilde{n}+1)$-element partition equilibria which $R$ prefers to all the equilibria in $\Gamma(b, s, r, 1)$.

Now, we show the following Lemma 2.

Lemma 2. Fix $b>0$ and suppose that $\tilde{n} \geq 2$. If $s / r<\eta(b)$, there exists $a(\tilde{n}+1)$-element partition equilibrium $((\hat{\sigma}, \hat{\rho}), \hat{f})$ such that $S$ always strictly prefers $((\hat{\sigma}, \hat{\rho}), \hat{f})$ to any equilibrium in a model with one-shot information transmission.

Now, we denote by $E U_{C S}^{S}$ the ex ante expected payoff of $S$ under the $\tilde{n}$-element partition equilibrium with $\left\{\tilde{a}_{\tilde{n}}, \ldots, \tilde{a}_{0}\right\}$ in a model with one-shot information transmission. We denote by $E \hat{U}^{S}(x)$ the ex ante expected payoff of $S$ under the $(\tilde{n}+1)$-element 
partition equilibrium $((\hat{\sigma}, \hat{\rho}), \hat{f})$ with $(\tilde{n}+1)$-element partition: $\left\{\left[\hat{a}_{\tilde{n}(b)+1}^{x}, \hat{a}_{\tilde{n}(b)}^{x}\right), \ldots\left[\hat{a}_{1}^{x}, \hat{a}_{0}^{x}\right]\right\}$ where $\hat{a}_{1}^{x} \equiv x \in\left(\underline{a}_{1}(s / r), 1\right)$.

Since the boundaries $\left\{\hat{a}_{\tilde{n}+1}^{x}, \ldots, \hat{a}_{1}^{x}\right\}$ almost coincides with the boundaries $\left\{\tilde{a}_{\tilde{n}}, \ldots, \tilde{a}_{0}\right\}$ when $\hat{a}_{1}^{x} \approx 1$, by taking $x$ suitably, we have $E \hat{U}^{S}(x)-E U_{C S}^{S}>0$.

Next, we show the following Lemma 3.

Lemma 3. Fix $b>0$ and suppose that $\tilde{n} \geq 2$. Then, there exists a positive value $\bar{\eta}(b)$ such that if $s / r<\bar{\eta}(b)$, there exists $\bar{x}, \underline{x} \in\left(\underline{a}_{1}(s / r), 1\right)$ such that

$$
E \hat{U}^{R}(\bar{x})>E U_{C S}^{R} \text { and } E \hat{U}^{R}(\underline{x})<E U_{C S}^{R} .
$$

Intuitively, $R$ seems to prefer the $(\tilde{n}+1)$-element partition with $\left\{\hat{a}_{\tilde{n}(b)+1}^{x}, \ldots, \hat{a}_{0}^{x}\right\}$ to the $\tilde{n}$-element partition with $\left\{\tilde{a}_{\tilde{n}}, \ldots, \tilde{a}_{0}\right\}$ since the former has more steps than the latter. We have already shown that if Condition $C$ holds, then there exists $x<1$ such that $\hat{W}(x)>E U_{C S}^{R}$. Fix $x$, then $\hat{W}(x)-E U_{C S}^{R}$ is increasing in $r$. Since, moreover, $w^{*}$ is decreasing and goes to 0 as $s$ goes to 0 , the expected payment $E\left[w^{*}\right]$ is also decreasing and goes to 0 as $s$ goes to 0 . Thus, if $r$ is large enough relative to $s$, then there exists $\bar{x}$ such that $E \hat{U}^{R}(\bar{x})>E U_{C S}^{R}$.

Now, fix $s$ and $r$. Then, $\hat{W}(x)-E U_{C S}^{R}$ goes to 0 as $x$ goes to 1 since $\left\{\hat{a}_{\tilde{n}+1}^{x}, \ldots, \hat{a}_{1}^{x}\right\}$ converges to $\left\{\tilde{a}_{\tilde{n}}, \ldots, \tilde{a}_{0}\right\}$ as $x$ goes to 1 . Nevertheless, $w^{*}$ goes to $s \cdot u^{S}\left(y^{R}(1), 1, b\right)-s$. $u^{S}\left(\bar{y}\left(\tilde{a}_{1}, 1\right), 1, b\right)>0$ and $\operatorname{Pr}\left(m_{1}<x\right) \approx 1$ as $x$ goes to $1 .{ }^{22}$ This means that $E\left[w^{*}\right]$ goes to $s \cdot u^{S}\left(y^{R}(1), 1, b\right)-s \cdot u^{S}\left(\bar{y}\left(\tilde{a}_{1}, 1\right), 1, b\right)>0$ as $x$ goes to 1 . Hence, there always exists $\underline{x} \approx 1$ such that $E \hat{U}^{R}(\underline{x})<E U_{C S}^{R}$.

Finally, we complete the proof of Proposition 13 by demonstrating that if $r$ is large enough relative to $s$, then we can take $x \in\left(\underline{a}_{1}(s / r), 1\right)$ such that

$$
E \hat{U}^{R}(x)>E U_{C S}^{R} \text { and } E \hat{U}^{S}(x)>E U_{C S}^{S} .
$$

The formal proof of Lemma 2-3, and Proposition 13 can be found in Appendix H-J.

\section{Conclusion}

In this paper, we studied multistage information transmission with voluntary monetary transfer in the framework of the CS model. We have shown that multistage information transmission with voluntary monetary transfer can lead to more informative equilibrium outcomes than those in the CS model. Moreover, we have shown that under multistage information transmission with voluntary monetary transfer, there exists an equilibrium whose outcome Pareto-dominates all the equilibrium outcomes in the CS model.

We have provided an upper bound (not necessarily the least upper bound) of $R^{\prime} \mathrm{s}$ ex ante expected payoff under partition equilibria. Moreover, we have provided a sufficient condition for this upper bound to be approximated by an $R$ 's equilibrium payoff. Consequently, we show that when the length of the communication round

\footnotetext{
${ }^{22}$ For details, see Appendix G.
} 
is high and $R$ places greater importance on the project than $S$ does, $R$ can obtain higher ex ante expected payoff under effective $T$-period communication than that under delegation and arbitration.

A full characterization of all equilibria in our model remains an open question.

\section{Appendix A Proof of Proposition 2}

Fix a pure strategy equilibrium under a direct contract $(\Theta, \omega)$. Then, the existence of a family of sets $\left\{\mathcal{I}_{\lambda}\right\}_{\lambda \in \Lambda}$ that satisfies the conditions 1-3 in Definition 2 is trivial. Hence, we have only to make sure that $\mathcal{I}_{\lambda}$ is convex for each $\lambda \in \Lambda$. First, we show that the $R$ 's strategy regarding the project, $y: \Theta \rightarrow Y$, satisfies the following property.

Lemma 4. In a pure strategy equilibrium under a direct contract $(\Theta, \omega)$, the receiver's strategy regarding the project, $y(\theta)$, is nondecreasing.

Proof of Lemma 4. From $S^{\prime}$ s incentive compatibility condition, for any $\theta, \theta^{\prime} \in \Theta$,

$$
\begin{aligned}
& u^{S}(y(\theta), \theta, b)+\omega(\theta) \geq u^{S}\left(y\left(\theta^{\prime}\right), \theta, b\right)+\omega\left(\theta^{\prime}\right), \text { and } \\
& u^{S}\left(y\left(\theta^{\prime}\right), \theta^{\prime}, b\right)+\omega\left(\theta^{\prime}\right) \geq u^{S}\left(y(\theta), \theta^{\prime}, b\right)+\omega(\theta) .
\end{aligned}
$$

This implies that

$$
u^{S}(y(\theta), \theta, b)-u^{S}\left(y\left(\theta^{\prime}\right), \theta, b\right) \geq u^{S}\left(y(\theta), \theta^{\prime}, b\right)-u^{S}\left(y\left(\theta^{\prime}\right), \theta^{\prime}, b\right)
$$

Since we suppose that $u_{1,2}^{S}>0$, we have $y(\theta) \geq y\left(\theta^{\prime}\right)$ for $\theta>\theta^{\prime}$.

From this lemma, we have the following lemma.

Lemma 5. In a pure strategy equilibrium under a direct contract $(\Theta, \omega)$, if $y(\underline{\theta})=y(\bar{\theta})$ for $\underline{\theta}<\bar{\theta}$, then $y(\underline{\theta})=y(\theta)=y(\bar{\theta})$ for all $\theta \in[\underline{\theta}, \bar{\theta}]$. Moreover, $\omega(\underline{\theta})=\omega(\theta)=\omega(\bar{\theta})$ for all $\bar{\theta} \in[\underline{\theta}, \bar{\theta}]$.

Lemma 5 implies the convexity of $\mathcal{I}_{\lambda}$.

\section{Appendix B Proof of Proposition 3}

We now suppose that there exists a fully separating equilibrium $\xi_{F}$. Let $\left(\Theta, \omega_{F}\right)$ be a direct contract under which there exists a pure strategy equilibrium that is outcome equivalent to $\xi_{F}$. Let $y(\theta)$ be the $R^{\prime}$ s equilibrium strategy under $\left(\Theta, \omega_{F}\right)$. Since $R$ learns the true state eventually under $\xi_{F}, y(\theta)=y^{R}(\theta)$ in the pure strategy equilibrium under $\left(\Theta, \omega_{F}\right)$. When truth telling is a best response for $S$, it is necessary to satisfy the condition:

$$
s \cdot u^{S}\left(y^{R}(\theta), \theta, b\right)+\omega(\theta) \geq s \cdot u^{S}\left(y^{R}\left(\theta^{\prime}\right), \theta, b\right)+\omega\left(\theta^{\prime}\right) \text { for all } \theta^{\prime} \neq \theta .
$$

From the first-order condition, we have the differential equation

$$
\frac{d}{d \theta} \omega(\theta)=-s \cdot u_{1}^{S}\left(y^{R}(\theta), \theta, b\right) \frac{d}{d \theta} y^{R}(\theta)
$$


Since $u_{1}^{S}\left(y^{R}(\theta), \theta, b\right)>0$ and $y^{\prime R}(\theta) \equiv \frac{d}{d \theta} y^{R}(\theta)>0$, S's incentive compatibility condition requires that

$$
\omega(\theta)=\omega(1)+\int_{\theta}^{1} s \cdot u_{1}^{S}\left(y^{R}(z), z, b\right) y^{\prime R}(z) d z .
$$

From the condition (13), the compensation schedule that induces full revelation is strictly decreasing in $\theta$. Finally, we show that $R$ 's payment strategy satisfing the condition (13) cannot be optimal for her. Let $\tilde{\mathbf{m}}$ be a sequence of messages that belongs to $\operatorname{supp} \mu_{\xi_{F}}(\cdot \mid \theta=0)$. Since $\sum_{t=1}^{T} w_{t}(\tilde{\mathbf{m}})=\omega(0)>0$ and $w_{T}(\tilde{\mathbf{m}})=0$, there exists $\tilde{t} \in$ $\{1, \ldots, T-1\}$ such that $w_{\tilde{t}}(\tilde{\mathbf{m}})>0$ and $w_{t}(\tilde{\mathbf{m}})=0$ for all $t \in\{\tilde{t}+1, \ldots, T\} .{ }^{23}$ Since $\omega(0)$ is the maximum value of $\omega(\theta)$, under the given equilibrium $\xi_{F}$, there exists an on-the-path history $\tilde{h} \in H^{(\tilde{t}, 2)}$, where $R$ pays a positive amount of money to $S$ at this history and never pays money after this history; $\rho(\tilde{h})>0$ and $\rho\left(\tilde{h}^{+}\right)=0$ for any $\tilde{h}^{+} \in\left\{h \in \cup_{t=\tilde{t}+1}^{T} H^{(t, 2)}: h\right.$ is consistent with $\tilde{h}$ and on-the-path history $\}$. Note that given $\tilde{\mathbf{m}}$, the history $\tilde{h}$ occurs with probability one by the assumption that we suppose that $R$ 's strategy is a pure strategy. Since $\xi_{F}$ is a full separating equilibrium, $R$ chooses $y^{R}(0)$ after $\tilde{h}$. Therefore, she has no incentive to pay a positive amount of money at $\tilde{h}$. For all $\mathbf{m} \in \operatorname{supp} \mu_{\xi_{F}}(\cdot \mid \theta=0)$, the same is true. This means that the given $R^{\prime}$ s payment strategy does not satisfy the equilibrium condition. $\diamond$

\section{Appendix C Proof of Proposition 8}

Fix an equilibrium $\xi$. Let $(\Theta, \omega)$ be a direct contract under which there exists a pure strategy equilibrium that is outcome equivalent to $\xi$. Let $y(\theta)$ be the $R^{\prime}$ 's equilibrium strategy under $(\Theta, \omega)$. We have already shown that $\xi$ is a partition equilibrium in the proof of Proposition 2. Let $\hat{\Theta}$ be the set of the boundaries of equilibrium partition. First, we show the following Lemma 6.

Lemma 6. If there exists open intervals $\left(\theta_{k+1}, \theta_{k}\right)$ and $\left(\theta_{j}, \theta_{j-1}\right)$ such that $\theta_{k+1}<\theta_{k} \leq \theta_{j}<$ $\theta_{j-1}$ and $\left(\theta_{k+1}, \theta_{k}\right),\left(\theta_{j}, \theta_{j-1}\right) \subset \hat{\Theta}$, then

- $\omega$ is strictly decreasing in $\theta$ over $\left(\theta_{k+1}, \theta_{k}\right)$ and $\left(\theta_{j}, \theta_{j-1}\right)$, and

- $\lim _{\theta \uparrow \theta_{k}} \omega(\theta) \equiv \underline{\omega}>\bar{\omega} \equiv \lim _{\theta \downarrow \theta_{j}} \omega(\theta)$.

Proof of Lemma 6. First, we show that $\omega$ is strictly decreasing in $\theta$ over $\left(\theta_{k+1}, \theta_{k}\right)$ and $\left(\theta_{j}, \theta_{j-1}\right)$. For all $\theta \in\left(\theta_{k+1}, \theta_{k}\right)$, the truth telling to be a best response requires that

$$
s \cdot u^{S}\left(y^{R}(\theta), \theta, b\right)+\omega(\theta) \geq s \cdot u^{S}\left(y^{R}\left(\theta^{\prime}\right), \theta, b\right)+\omega\left(\theta^{\prime}\right) \quad \text { for all } \theta^{\prime}, \theta \in\left(\theta_{k+1}, \theta_{k}\right) .
$$

The first-order condition for $S$ results in the differential equation

$$
\frac{d}{d \theta} \omega(\theta)=-s \cdot u_{1}^{S}\left(y^{R}(\theta), \theta, b\right) \frac{d}{d \theta} y^{R}(\theta)
$$

\footnotetext{
${ }^{23}$ Since $R$ cannot obtain additional information about $\theta$ after stage 2 in period $T$, she has no incentive to choose $w^{T}>0$.
} 
Since $u_{1}^{S}\left(y^{R}(\theta), \theta, b\right)>0$ and $y^{\prime R}(\theta) \equiv \frac{d}{d \theta} y^{R}(\theta)>0$, S's incentive compatibility condition requires that

$$
\omega(\theta)=\omega\left(\theta_{k}\right)+\int_{\theta}^{\theta_{k}} s \cdot u_{1}^{S}\left(y^{R}(z), z, b\right) y^{\prime R}(z) d z .
$$

The same argument holds for interval $\left(\theta_{j}, \theta_{j-1}\right)$. Hence, we have

$$
\omega(\theta)=\omega\left(\theta_{j-1}\right)+\int_{\theta}^{\theta_{j-1}} s \cdot u_{1}^{S}\left(y^{R}(z), z, b\right) y^{\prime R}(z) d z .
$$

From the conditions (14) and (15), the compensation schedule that induces the full revelation is strictly decreasing in $\theta$ over $\left(\theta_{k+1}, \theta_{k}\right)$ and $\left(\theta_{j}, \theta_{j-1}\right)$.

To simplify the proof, we now suppose that there exists no open interval $(\underline{\theta}, \bar{\theta}) \subset$ $\left(\theta_{k}, \theta_{j}\right)$ such that $(\underline{\theta}, \bar{\theta}) \subset \hat{\Theta}$. Since we now consider the uniform-quadratic case, the equilibrium payoffs of $S$ of type $\theta_{k}$ and $\theta_{j}$ are $-s b^{2}+\underline{\omega}$ and $-s b^{2}+\bar{\omega}$, respectively. Moreover, under the uniform-quadratic assumption, if $\left[\theta^{\prime}, \theta^{\prime \prime}\right]$ is an element of equilibrium partition $^{24}$ such that $\theta^{\prime}<\theta^{\prime \prime}$, then

$$
\begin{aligned}
\lim _{\theta \downarrow \theta^{\prime}} s \cdot u^{S}(y(\theta), \theta, b) & +\omega^{\prime}=s \cdot u^{S}\left(y\left(\theta^{\prime}\right), \theta^{\prime}, b\right)+\omega^{\prime} \\
& >s \cdot u^{S}\left(y\left(\theta^{\prime \prime}\right), \theta^{\prime \prime}, b\right)+\omega^{\prime \prime}=\lim _{\theta \uparrow \theta^{\prime \prime}} s \cdot u^{S}(y(\theta), \theta, b)+\omega^{\prime \prime}
\end{aligned}
$$

where $\omega^{\prime}=\omega^{\prime \prime}=\omega(\theta)$ for $\theta \in\left(\theta^{\prime}, \theta^{\prime \prime}\right)$ since $s \cdot u^{S}\left(y\left(\theta^{\prime}\right), \theta^{\prime}, b\right)=s\left(\left(\theta^{\prime}+\theta^{\prime \prime}\right) / 2-\theta^{\prime}-b\right)^{2}$ and $s \cdot u^{S}\left(y\left(\theta^{\prime \prime}\right), \theta^{\prime \prime}, b\right)=s\left(\left(\theta^{\prime}+\theta^{\prime \prime}\right) / 2-\theta^{\prime \prime}-b\right)^{2}$. We have

$$
s \cdot u^{S}\left(\left(\theta^{\prime}+\theta^{\prime \prime}\right) / 2, \theta^{\prime}, b\right)>s \cdot u^{S}\left(\left(\theta^{\prime}+\theta^{\prime \prime}\right) / 2, \theta^{\prime \prime}, b\right) .
$$

From the conditions (14)-(16), we can conclude that $s \cdot u^{S}(y(\theta), \theta, b)+\omega(\theta)$ is strictly decreasing in $\theta$ over $\left(\theta_{k+1}, \theta_{j-1}\right) \cap \hat{\Theta}$. Therefore, we have

$$
\begin{aligned}
\lim _{\theta \uparrow \theta_{k}} s \cdot u^{S}(y(\theta), \theta, b)+\omega(\theta) & =-s b^{2}+\underline{\omega} \\
& >-s b^{2}+\bar{\omega}=\lim _{\theta \downarrow \theta_{j}} s \cdot u^{S}(y(\theta), \theta, b) .
\end{aligned}
$$

This completes the proof of Lemma 6.

Now we suppose that there exists an open interval $\left(\theta_{k+1}, \theta_{k}\right) \subset \hat{\Theta}$. Lemma 6 implies that for almost every $\hat{\theta} \in\left(\theta_{k+1}, \theta_{k}\right)$, there is no $\tilde{\theta} \in \Theta \backslash\left(\theta_{k+1}, \theta_{k}\right)$ such that $\omega(\tilde{\theta})=\omega(\hat{\theta})$. This means that $R$ surely reaches a history such that she pays positive amount of money to $S$ even though she has already known the $S$ 's type. This holds almost every $\theta \in\left(\theta_{k+1}, \theta_{k}\right)$. Hence, we can conclude that there exists no open interval $\left(\theta_{k+1}, \theta_{k}\right)$ such that $\left(\theta_{k+1}, \theta_{k}\right) \subset \hat{\Theta}$.

Next, we show that the cardinality of $\hat{\Theta}$ is finite. We prove this by contradiction. Now, suppose that the cardinality of $\hat{\Theta}$ is countably infinite. Then, we can take an infinite sequence $\left\{\theta_{n}\right\}_{n \in \mathbb{N}}$ such that

\footnotetext{
${ }^{24}$ The same argument holds for the cases of $\left[\theta^{\prime}, \theta^{\prime \prime}\right),\left(\theta^{\prime}, \theta^{\prime \prime}\right]$ and $\left(\theta^{\prime}, \theta^{\prime \prime}\right)$
} 
- $\lim _{n \rightarrow \infty} \theta_{n}=L$ for some $L \in \hat{\Theta}$,

- $\Upsilon \notin \hat{\Theta}$ for any $\Upsilon \in\left(\inf \left\{\theta_{n}\right\}_{n \in \mathbb{N}}, \sup \left\{\theta_{n}\right\}_{n \in \mathbb{N}}\right) \backslash\left\{\theta_{n}\right\}_{n \in \mathbb{N}}$, and

- $\sup \left\{\theta_{n}\right\}_{n \in \mathbb{N}}-\inf \left\{\theta_{n}\right\}_{n \in \mathbb{N}}<4 b$.

Since $\sup \left\{\theta_{n}\right\}_{n \in \mathbb{N}}-\inf \left\{\theta_{n}\right\}_{n \in \mathbb{N}}<4 b$, we have $\theta_{n^{\prime}}-\theta_{n}<4 b$ for $n<n^{\prime}$.

Define $\underline{o}, o$ and $\bar{o}$ as elements of $\left\{\theta_{n}\right\}_{n \in \mathbb{N}}$ such that $\underline{o}<o<\bar{o}$ and $\theta \notin \hat{\Theta}$ for any $\theta \in(\underline{o}, \bar{o}) \backslash\{o\}$. Let $\omega_{\underline{o}}$ be $\omega\left(\theta_{\underline{o}}\right)$ for $\theta_{\underline{o}} \in(\underline{o}, o)$. Let $\omega_{\bar{o}}$ be $\omega\left(\theta_{\bar{o}}\right)$ for $\theta_{\bar{o}} \in(o, \bar{o})$. Then, we have $s \cdot u^{S}\left(y\left(\theta_{o}\right), o, b\right)+\omega_{\underline{o}}=s \cdot u^{S}\left(y\left(\theta_{\bar{o}}\right), o, b\right)+\omega_{\bar{o}}$. Since $\bar{o}-o<o-\underline{o}+4 b$, we have $\omega_{\underline{o}}-\omega_{\bar{o}}=s \cdot u^{S}\left(y\left(\theta_{\bar{o}}\right), o, b\right)-s \cdot u^{S}\left(y\left(\theta_{\underline{o}}\right), o, b\right)>0$. Therefore, the image of $\omega$ the subset $\left(\inf \left\{\theta_{n}\right\}_{n \in \mathbb{N}}, \sup \left\{\theta_{n}\right\}_{n \in \mathbb{N}}\right) \subset \Theta$ under $\omega$ is a countably infinite set. Now, suppose that $\#\left\{\hat{\Theta} \backslash\left\{\theta_{n}\right\}_{n \in \mathbb{N}}\right\}<\infty$. Then, there exists an open interval $(\underline{v}, \bar{v}) \subset\left(\inf \left\{\theta_{n}\right\}_{n \in \mathbb{N}}, \sup \left\{\theta_{n}\right\}_{n \in \mathbb{N}}\right)$ such that

- $\omega\left(v^{\prime}\right)=\omega\left(v^{\prime \prime}\right)$ and $y\left(v^{\prime}\right)=y\left(v^{\prime \prime}\right)$ for all $v^{\prime}, v^{\prime \prime} \in(\underline{v}, \bar{v})$, and

- $\omega\left(\tilde{v}^{\prime}\right) \neq \omega(\tilde{v})$ and $y\left(v^{\prime}\right) \neq y(\tilde{v})$ for any $\tilde{v} \notin(\underline{v}, \bar{v})$.

This means that if the $S^{\prime}$ s type belongs to $(\underline{v}, \bar{v})$, then $R$ surely reaches a history such that she pays positive amount of money to $S$ even though she does not obtain additional information in the future. Hence, under the given strategy profile, $R$ reaches such a history with positive probabilities: $\operatorname{Pr}(\theta \in(\underline{v}, \bar{v}))$. Hence, we can conclude that there must be $\#\left\{\hat{\Theta} \backslash\left\{\theta_{n}\right\}_{n \in \mathbb{N}}\right\}=+\infty$. This implies that we can take an infinite sequence $\left\{\tilde{\theta}_{\zeta}\right\}_{\zeta \in \mathbb{N}}$ such that

- $\lim _{\zeta \rightarrow \infty} \tilde{\theta}_{\zeta}=\Psi$ for some $\Psi \in \Theta \backslash\left(\inf \left\{\theta_{n}\right\}_{n \in \mathbb{N}}, \sup \left\{\theta_{n}\right\}_{n \in \mathbb{N}}\right)$;

- $\hat{\Upsilon} \notin \hat{\Theta}$ for any $\hat{\Upsilon} \in\left(\inf \left\{\tilde{\theta}_{\zeta}\right\}_{\zeta \in \mathbb{N}}, \sup \left\{\tilde{\theta}_{\zeta}\right\}_{\zeta \in \mathbb{N}}\right) \backslash\left\{\tilde{\theta}_{\zeta}\right\}_{\zeta \in \mathbb{N}} ;$

- $\sup \left\{\tilde{\theta}_{\zeta}\right\}_{\zeta \in \mathbb{N}}-\inf \left\{\tilde{\theta}_{\zeta}\right\}_{\zeta \in \mathbb{N}}<4 b$.

In the same way as in the proof of Lemma 6, we can make sure that $\{\omega(\theta): \theta \in$ $\left.\left(\inf \left\{\theta_{n}\right\}_{n \in \mathbb{N}}, \sup \left\{\theta_{n}\right\}_{n \in \mathbb{N}}\right)\right\} \cap\left\{\omega(\theta): \theta \in\left(\inf \left\{\tilde{\theta}_{\zeta}\right\}_{\zeta \in \mathbb{N}}, \sup \left\{\tilde{\theta}_{\zeta}\right\}_{\zeta \in \mathbb{N}}\right)\right\}=\emptyset$. Therefore, under the given strategy profile, $R$ reaches a history such that she pays positive amount of money to $S$ even though she does not obtain additional information in the future, even if $\#\left\{\hat{\Theta} \backslash\left\{\theta_{n}\right\}_{n \in \mathbb{N}}\right\}=+\infty$. This means that the given $R^{\prime}$ s payment strategy does not satisfy the equilibrium condition. Therefore, the cardinality of $\hat{\Theta}$ must be finite. $\diamond$

\section{Appendix D Proof of Proposition 9}

We make sure that $-16 r b^{3} / 3$ is an upper bound (not necessarily the least upper bound) of $R^{\prime}$ s ex ante expected payoff under partition equilibria. Fix an equilibrium $\xi$, and a ( $S^{\prime}$ s incentive compatible) direct contract $(\Theta, \omega)$, which is outcome equivalent to $\xi$. Suppose that $\omega(\theta)$ is decreasing. Then, for $R^{\prime}$ s payment strategy to be optimal, there must be two elements equilibrium partition, $\left[0, a^{\prime}\right)$ and $\left[a^{\prime}, a^{\prime \prime}\right)$, where $w\left(\theta^{\prime}\right)=w\left(\theta^{\prime \prime}\right)$ for $\theta^{\prime} \in\left[0, a^{\prime}\right)$ and $\theta^{\prime \prime} \in\left[a^{\prime}, a^{\prime \prime}\right)$. This means that the boundary $a^{\prime}$ must coincide with the boundary of the 2-element partition (CS) equilibrium where the state space is $\left[0, a^{\prime \prime}\right)$. If 
$a^{\prime \prime}<4 b$, then $S^{\prime}$ s indifferent condition $s \cdot u^{S}\left(\bar{y}\left(0, a^{\prime}\right), a^{\prime}, b\right)-s \cdot u^{S}\left(\bar{y}\left(a^{\prime}, a^{\prime \prime}\right), a^{\prime}, b\right)=0$ has no solution. Therefore, it must be satisfied that $a^{\prime \prime} \geq 4 b$ when $\omega(\theta)$ is decreasing.

Now we suppose that $\omega(\theta)$ is nondecreasing. Then, there are two elements of equilibrium partition, $\left[a, a^{\prime}\right]$ and $\left(a^{\prime}, a^{\prime \prime}\right]$, where $w\left(\theta^{\prime}\right)<w\left(\theta^{\prime \prime}\right)$ for $\theta^{\prime} \in\left[a, a^{\prime}\right]$ and $\theta^{\prime \prime} \in$ $\left(a^{\prime}, a^{\prime \prime}\right]$. Now, we allow the case where $a=a^{\prime}$, this means that $S$ tells the truth when $\theta=a$. For $S^{\prime}$ s indifferent condition, it must be satisfied that

$$
U^{S}\left(y\left(\theta^{\prime}\right), \theta^{\prime}, \omega\left(\theta^{\prime}\right)\right)=U^{S}\left(y\left(\theta^{\prime \prime}\right), \theta^{\prime \prime}, \omega\left(\theta^{\prime \prime}\right)\right)
$$

This implies that

$$
\omega\left(\theta^{\prime \prime}\right)-\omega\left(\theta^{\prime}\right)=s\left[\left(\frac{a^{\prime}+a^{\prime \prime}}{2}-a^{\prime}-b\right)^{2}-\left(\frac{a+a^{\prime}}{2}-a^{\prime}-b\right)^{2}\right]>0 .
$$

Therefore, it must be satisfied that $a^{\prime}-a+4 b<a^{\prime \prime}-a^{\prime 25}$. Hence, we conclude that $R^{\prime}$ s ex ante expected payoff under $(\Theta, \omega)$ is lower than $-16 r b^{3} / 3$. This implies that $-16 r b^{3} / 3$ is an upper bound (not necessarily the least upper bound) of $R^{\prime}$ s ex ante expected payoff under partition equilibria. $\diamond$

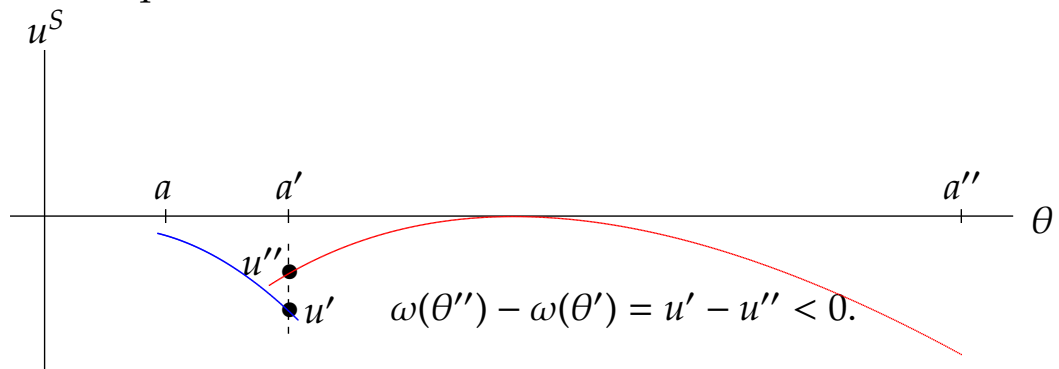

Figure 5: $a^{\prime \prime}-a^{\prime}<a^{\prime}-a+4 b$

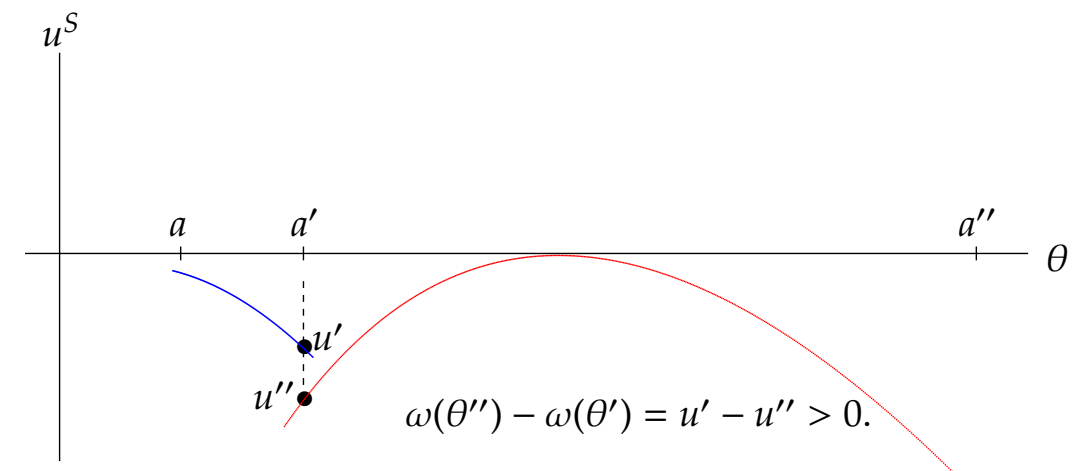

Figure 6: $a^{\prime \prime}-a^{\prime}>a^{\prime}-a+4 b$

\footnotetext{
${ }^{25}$ See Figure 5 and Figure 6.
} 


\section{Appendix E Proof of Proposition 10}

First, we now make sure of the optimality of $S^{\prime}$ s strategy. At history $h^{(t, 1)}$ in which $w_{t^{\prime}}<w_{t^{\prime}}^{*}$ or $m_{t^{\prime}} \geq a_{t^{\prime}}$ for some $t^{\prime}<t$, any type of $S$ send a message randomly according to the same distribution, a uniform distribution over $[0,1]$. Therefore, there is no profitable deviation for $S$ at such a history.

At history $h^{(1,1)}$ or $h^{(t, 1)}$ in which $m_{t^{\prime}}<a_{t^{\prime}}$ and $w_{t^{\prime}} \geq w_{t^{\prime}}^{*}$ for all $t^{\prime}<t$, if $S$ of type $\theta$ sends $m_{t} \geq a_{t}$, then he will obtain $-s\left(\frac{a_{t}+a_{t-1}}{2}-(\theta+b)\right)^{2}$ in the future. Otherwise, the continuation payoff of $S$ can be $-s\left(\frac{a_{\tilde{t}+1}+a_{\tilde{t}}}{2}-(\theta+b)\right)^{2}+\sum_{l=t}^{\tilde{t}} w_{l}^{*}$ for $\tilde{t} \in\{t, \ldots, T\}$. By $a_{t}$ and $w_{t}^{*}$ defined above, it is easy to verify that for any $\tilde{t} \in\{t, \ldots, T\}$,

$$
\begin{array}{lr}
-s\left(\frac{a_{t}+a_{t-1}}{2}-(\theta+b)\right)^{2}>-s\left(\frac{a_{\tilde{t}+1}+a_{\tilde{t}}}{2}-(\theta+b)\right)^{2}+\sum_{l=t}^{\tilde{t}} w_{l}^{*} \quad \text { for any } \theta>a_{t}, \\
-s\left(\frac{a_{t}+a_{t-1}}{2}-(\theta+b)\right)^{2}<-s\left(\frac{a_{\tilde{t}+1}+a_{\tilde{t}}}{2}-(\theta+b)\right)^{2}+\sum_{l=t}^{\tilde{t}} w_{l}^{*} & \text { for any } \theta \in\left[a_{\tilde{t}+1}, a_{\tilde{t}}\right), \\
-s\left(\frac{a_{t}+a_{t-1}}{2}-(\theta+b)\right)^{2}=-s\left(\frac{a_{t+1}+a_{t}}{2}-(\theta+b)\right)^{2}+w_{t}^{*} & \text { for } \theta=a_{t} .
\end{array}
$$

Moreover, for $\theta=a_{t}$,

$$
\max \left\{-s\left(\frac{a_{\tilde{t}+1}+a_{\tilde{t}}}{2}-(\theta+b)\right)^{2}+\sum_{l=t}^{\tilde{t}} w_{l}^{*}\right\}_{\tilde{t} \in\{t, \ldots, T\}}=-s\left(\frac{a_{t+1}+a_{t}}{2}-(\theta+b)\right)^{2}+w_{t}^{*} .
$$

Since these (17)-(19) hold for any $t \in\{1, \ldots, T\}$, there is no profitable deviation for $S$ from $\xi_{T}$.

Next, we make sure of the optimality of $R^{\prime}$ s strategy. At any history $h^{T+1} \in H^{T+1}$, the posterior belief $f\left(\theta \mid h^{T+1} \equiv\left(h^{(T, 2)}, w_{T}\right)\right)=f\left(\theta \mid h^{(T, 2)}\right)$ is a uniform distribution supported on an interval whose mid-point is equal to $\frac{\min I\left(h^{T+1}\right)+\max I\left(h^{T+1}\right)}{2}$. Therefore, $y=\frac{\min I\left(h^{T+1}\right)+\max I\left(h^{T+1}\right)}{2}$ is an optimal project for $R$ at any $h^{T+1} \in H^{T+1}$.

At history $h^{(t, 2)}$ in which $w_{t^{\prime}}<w_{t^{\prime}}^{*}$ or $m_{t^{\prime}} \geq a_{t^{\prime}}$ for some $t^{\prime}<t, R$ has no chance to obtain additional information about $k$ in the future. Therefore, he must pay nothing to $S$ at such a history. At history $h^{(T-1,2)}$ in which he learns $\theta<a_{T-1}$ at the preceding stage $1, m_{t^{\prime}}<a_{t^{\prime}}$ and $w_{t^{\prime}} \geq w_{t^{\prime}}^{*}$ for all $t^{\prime}<T-1$ and $m_{T-1}<a_{T-1}$, if $R$ pays $w_{T-1} \geq w_{T-1^{\prime}}^{*}$ then he obtains $u_{T-1}^{*}\left(w_{T-1}\right)$ :

$$
\begin{aligned}
u_{T-1}^{*}\left(w_{T-1}\right) & =-w_{T-1}-r \sum_{i=T}^{T+1} \int_{a_{i}}^{a_{i-1}} \frac{1}{a_{T-1}}\left(\frac{a_{i}+a_{i-1}}{2}-\theta\right)^{2} d \theta \\
& =-w_{T-1}-r\left(\frac{\left(a_{T}\right)^{3}}{12 a_{T-1}}+\frac{\left(a_{T-1}-a_{T}\right)^{3}}{12 a_{T-1}}\right) \\
& =-w_{T-1}-r\left(b^{2}+\frac{\left(a_{T-1}\right)^{2}}{48}\right) .
\end{aligned}
$$

Clearly, $u_{T-1}^{*}\left(w_{T-1}\right)$ takes the maximum value $u_{T-1}^{*}$ at $w_{T-1}=w_{T-1}^{*}$. On the other hand, by paying $w_{T-1}<w_{T-1}^{*}$, he obtains $\bar{u}_{T-1}\left(w_{T-1}\right)$ : 


$$
\begin{aligned}
\bar{u}_{T-1}\left(w_{T-1}\right) & =-w_{T-1}-r \int_{0}^{a_{T-1}} \frac{1}{a_{T-1}}\left(\frac{a_{T-1}}{2}-\theta\right)^{2} d \theta \\
& =-w_{T-1}-r \frac{\left(a_{T-1}\right)^{2}}{12} .
\end{aligned}
$$

Clearly, $\bar{u}_{T-1}\left(w_{T-1}\right)$ takes the maximum value $\bar{u}_{T-1}$ at $w_{T-1}=0$. Therefore, paying $w_{T-1}^{*}$ is optimal for $R$ if and only if $u_{T-1}^{*} \geq \bar{u}_{T-1}$.

$$
u_{T-1}^{*} \geq \bar{u}_{T-1} \Longleftrightarrow r\left(\frac{\left(a_{T-1}\right)^{2}}{16}-b^{2}\right) \geq w_{T-1}^{*} .
$$

By making $a$ sufficiently close to 0 , the left-hand side of this inequality can be made to be as close to $r\left(\frac{1}{16}-b^{2}\right)$ as desired and the right-hand side of this inequality can be made to be as close to $\frac{s}{16}\{1+12\}\{1+4 b\}$ as desired. It is obvious that if $\frac{s}{r}<\frac{1-4 b}{1+12 b}$, there exists $\hat{a}(b, T)>0$ such that if $a<\hat{a}(b, T)$, then $u_{T-1}^{*}>\bar{u}_{T-1}$.

At history $h^{(t, 2)}, t \in\{1, \ldots, T-2\}$, in which he learns $\theta<a_{t}$ at the preceding stage 1 , $m_{t^{\prime}}<a_{t^{\prime}}$ and $w_{t^{\prime}} \geq w_{t^{\prime}}^{*}$ for all $t^{\prime}<t$ and $m_{t}<a_{t}$, if $R$ pays $w_{t} \geq w_{t^{\prime}}^{*}$, then he obtains $u_{t}^{*}\left(w_{t}\right)$ :

$$
\begin{aligned}
u_{t}^{*}\left(w_{t}\right) & =-w_{t}-\sum_{i=t+1}^{T} w_{i}^{*} \frac{a_{i}}{a_{i-1}}-r \sum_{i=t+1}^{T+1} \int_{a_{i}}^{a_{i-1}} \frac{1}{a_{t}}\left(\frac{a_{i}+a_{i-1}}{2}-\theta\right)^{2} d \theta \\
& =-w_{t}-\sum_{i=t+1}^{T} w_{i}^{*} \frac{a_{i}}{a_{i-1}}-r\left(\frac{a_{T-1} b^{2}}{a_{t}}+\frac{\left(a_{T-1}\right)^{3}}{48 a_{t}}+(T-1-t) \frac{(a)^{3}}{48 a_{t}}\right) .
\end{aligned}
$$

Clearly, $u_{t}^{*}\left(w_{t}\right)$ takes the maximum value $u_{t}^{*}$ at $w_{t}=w_{t}^{*}$. On the other hand, by paying $w_{t}<w_{t}^{*}$, he obtains $\bar{u}_{t}\left(w_{t}\right)$ :

$$
\begin{gathered}
\bar{u}_{t}\left(w_{t}\right)=-w_{t}-r \int_{0}^{a_{t}} \frac{1}{a_{t}}\left(\frac{a_{t}}{2}-k\right)^{2} d k \\
=-w_{t}-r \frac{\left(a_{t}\right)^{2}}{12} .
\end{gathered}
$$

Clearly, $\bar{u}_{t}\left(w_{t}\right)$ takes the maximum value $\bar{u}_{t}$ at $w_{t}=0$. Therefore, paying $w_{t}^{*}$ is optimal for $R$ if and only if $u_{t}^{*} \geq \bar{u}_{t}$.

$$
u_{t}^{*} \geq \bar{u}_{t} \Longleftrightarrow r\left(-\frac{a_{T-1} b^{2}}{a_{t}}-\frac{\left(a_{T-1}\right)^{3}}{48 a_{t}}-(T-1-t) \frac{(a)^{3}}{48 a_{t}}+\frac{\left(a_{t}\right)^{2}}{12}\right) \geq \sum_{i=t+1}^{T} w_{i}^{*} \frac{a_{i}}{a_{i-1}}+w_{t}^{*} .
$$

By making $a$ sufficiently close to 0 , the left-hand side of this inequality can be made to be as close to $r\left(\frac{1}{16}-b^{2}\right)$ as desired and the right-hand side of this inequality can be made to be as close to $\frac{s}{16}\{1+12\}\{1+4 b\}$ as desired. It is obvious that if $\frac{s}{r}<\frac{1-4 b}{1+12 b}$, there exists $\tilde{a}(b, T)>0$ such that if $a<\tilde{a}(b, T)$, then $u_{t}^{*}>\bar{u}_{t}$ for any $t \in\{1, \ldots, T-2\}$. Take $a<\min \left\{\frac{1+12 b}{T+1}, \frac{1-4 b}{T-1}, \hat{a}(b, T), \tilde{a}(b, T)\right\}$. Then, $\xi_{T}$ constitutes an equilibrium. $\diamond$ 


\section{Appendix F Proof of Proposition 11}

We now impose a condition, $a=\frac{1-(4 b+\varepsilon)}{T-1}$, on $\xi_{T}$. Since $a_{T-1}=4 b+\varepsilon \in(4 b, 1)$, it must be satisfied that $\varepsilon \in(0,1-4 b)$. Moreover, it holds that $a=\frac{1-(4 b+\varepsilon)}{T-1}<\frac{1-4 b}{T-1}<\frac{1+4 b}{T-3}$. Therefore, if $a<\frac{1+12 b}{T+1}, a_{i}$ and $w_{t}^{*}$ are well-defined. We now suppose that $T>\tilde{T}(b) \equiv \frac{1}{8 b}+\frac{1}{2}$, and then $a<\frac{1+12 b}{T+1}$ for any $\varepsilon \in(0,1-4 b)$. Let $\xi_{\varepsilon}$ be this modified strategy profile and system of beliefs. The following lemma shows that if $r$ is large relative to $s$, then $\xi_{\varepsilon}$ can be an equilibrium.

Lemma 7. Fix $b \in(0,1 / 4)$, and $T \geq \tilde{T}(b)$. Then, for any $\varepsilon \in(0,1-4 b)$, there exists $\underline{\eta}(b, T, \varepsilon)$ such that if $s / r<\underline{\eta}(b, T, \varepsilon)$, then $\xi_{\varepsilon}$ can be an equilibrium.

Proof of Lemma 7. It is obvious that the condition $a=\frac{1-(4 b+\varepsilon)}{T-1}$ only affects $R^{\prime}$ s optimal decision at $h^{(t, 2)}, t \in\{1, \ldots, T-1\}$, in which he learns $\theta<a_{t}$ at the preceding stage 1, $m_{t^{\prime}}<a_{t^{\prime}}$ and $w_{t^{\prime}} \geq w_{t^{\prime}}^{*}$ for all $t^{\prime}<t-1$ and $m_{t}<a_{t}$. Therefore, we have only to make sure whether the inequalities (20) and (21) hold.

Since $a_{T-1}=4 b+\varepsilon>4 b$, we have $\frac{\left(a_{T-1}\right)^{2}}{16}-b^{2}>0$. Therefore, we have

$$
\begin{aligned}
u_{T-1}^{*} \geq \bar{u}_{T-1} & \Longleftrightarrow \\
& \frac{s}{r} \leq \frac{\left(a_{T-1}\right)^{2}-16 b^{2}}{\{1+12 b-a(T+1)\}\{1+4 b+a(3-T)\}} .
\end{aligned}
$$

The left-hand side of the inequality (21) can be simplified into

$$
r \frac{a_{T-1}}{a_{t}}\left\{\frac{4\left(a_{t}\right)^{3}-\left(a_{T-1}\right)^{3}-(T-1-t) a^{3}}{48 a_{T-1}}-\frac{a_{T-1}}{a_{t}} b^{2}\right\} .
$$

Since $a_{t}=a_{T-1}+(T-1-t) a$, we have

$$
\frac{4\left(a_{t}\right)^{3}-\left(a_{T-1}\right)^{3}-(T-1-t) a^{3}}{48 a_{T-1}}>\frac{\left(a_{t}\right)^{3}}{16 a_{T-1}}>\frac{\left(a_{t}\right)^{2}}{16}>b^{2}>\frac{a_{T-1}}{a_{t}} b^{2} .
$$

This implies that

$$
\frac{a_{T-1}}{a_{t}}\left\{\frac{4\left(a_{t}\right)^{3}-\left(a_{T-1}\right)^{3}-(T-1-t) a^{3}}{48 a_{T-1}}-\frac{a_{T-1}}{a_{t}} b^{2}\right\}>0 .
$$

Since, moreover, $w_{t}^{*}>0$, the right-hand side of the inequality (21) is higher than 0 . Therefore, we have

$$
\begin{aligned}
u_{t}^{*} \geq \bar{u}_{t} & \Longleftrightarrow \\
& \frac{s}{r}<\frac{\frac{a_{T-1}}{a_{t}}\left\{\frac{4\left(a_{t}\right)^{3}-\left(a_{T-1}\right)^{3}-(T-1-t) a^{3}}{48 a_{T-1}}-\frac{a_{T-1}}{a_{t}} b^{2}\right\}}{\frac{1}{s} \sum_{i=t+1}^{T} w_{i}^{*} \frac{a_{i}}{a_{i-1}}} .
\end{aligned}
$$

Note that the value of $\frac{w_{i}^{*}}{s}$ does not depend on $s$. Now we can conclude that there exists $\eta(b, T, \varepsilon)$ such that if $\frac{s}{r}<\underline{\eta}(b, T, \varepsilon)$, then the inequalities (22) and (23) hold and $\xi_{\varepsilon}$ constitutes an equilibrium. 
We denote by $E U^{R}(\varepsilon)$ the ex ante expected payoff of $R$ under a strategy profile $\xi_{\varepsilon}$.

$$
E U^{R}(\varepsilon)=r W(\varepsilon)-\sum_{i=1}^{T} w_{i}^{*} \frac{a_{i}}{a_{i-1}} .
$$

$r W(\varepsilon)$ denotes the expected revenue from the project under $\xi_{\varepsilon}$ :

$$
\begin{aligned}
r W(\varepsilon) & =-r \sum_{i=1}^{T+1} \int_{a_{i}}^{a_{i-1}}\left(\frac{a_{i}+a_{i-1}}{2}-\theta\right)^{2} d \theta \\
& =r\left[-(4 b+\varepsilon) b^{2}-\frac{(4 b+\varepsilon)^{3}}{48}-\frac{1}{48} \frac{\{1-(4 b+\varepsilon)\}^{3}}{(T-1)^{2}}\right] .
\end{aligned}
$$

There exists $\varepsilon(b, d, r)>0$ such that if $\varepsilon \in(0, \varepsilon(b, d, r))$, then

$$
r\left[-(4 b+\varepsilon) b^{2}-\frac{(4 b+\varepsilon)^{3}}{48}\right]>-\frac{16}{3} r b^{3}-d .
$$

This implies that for any $\varepsilon \in(0, \varepsilon(b, d, r))$, there exists $\bar{T}(b, \varepsilon, d, r)$ such that for any $T \geq \bar{T}(b, \varepsilon, d, r)$,

$$
r W(\varepsilon)>-\frac{16}{3} r b^{3}-d .
$$

Recall that $w_{i}^{*}$ is linear increasing in $s$ for all $i \in\{1, \ldots, T\}$. Suppose that $T \geq$ $\bar{T}(b, \varepsilon, d, r)$. Then, for any $\varepsilon \in(0, \varepsilon(b, d, r))$, there exists $\hat{\eta}(b, T, \varepsilon, d, r)$ such that if $s<$ $\hat{\eta}(b, T, \varepsilon, d, r)$, we have $E U^{R}(\varepsilon)>-16 r b^{3} / 3-d$.

Suppose that $T \geq \bar{T}(b, d, r)$ and $\varepsilon \in(0, \varepsilon(b, d, r))$. By Lemma 7 , it is obvious that if $\frac{s}{r}<\eta(b, T, \varepsilon)$, then $\xi_{\varepsilon}$ constitutes an equilibrium. Therefore, if $s<\tilde{\eta}(b, T, \varepsilon, d, r) \equiv$ $\min \{\hat{\eta}(\bar{b}, T, \varepsilon, d, r), r \cdot \eta(b, T, \varepsilon)\}$, the strategy profile $\xi_{\varepsilon}$ constitutes an equilibrium under which $\operatorname{EU}^{R}(\varepsilon)>-1 \overline{6 r} b^{3} / 3-d$.

We define $\bar{T}(b, d, r)$ and $\mathbb{E}(b, d, r)$ as follows.

$$
\begin{aligned}
& \bar{T}(b, d, r) \equiv \max \left\{\min _{\varepsilon \in(0, \varepsilon(b, d, r))} \bar{T}(b, \varepsilon, d, r), \tilde{T}(b)\right\}, \text { and } \\
& \mathbb{E}(b, d, r) \equiv\{\varepsilon \in(0, \varepsilon(b, d, r)): \bar{T}(b, \varepsilon, d, r)=\bar{T}(b, d, r)\} .
\end{aligned}
$$

Define $\eta(b, T, d, r)$ as follows:

$$
\underline{\eta}(b, T, d, r) \equiv \sup _{\varepsilon \in \mathbb{E}(b, d, r)} \tilde{\eta}(b, T, \varepsilon, d, r) .
$$

This completes the proof. $\diamond$

\section{Appendix G Proof of Proposition 12}

Let $\mathcal{H}$ be the set of all histories where $R$ makes a decision, $\mathcal{H} \equiv\left\{\bigcup_{t=1}^{T} H^{(t, 2)}\right\} \cup H^{T+1}$. We denote by $I(h)$ the closure of the set $\{\theta \in \Theta: f(\theta \mid h \in \mathcal{H})>0\}$. Under the belief system $\hat{f}$, we have $I\left(h^{T+1}\right) \in\left\{\left[\hat{a}_{\tilde{n}+1}, \hat{a}_{\tilde{n}}\right], \ldots\left[\hat{a}_{1}, \hat{a}_{0}\right],\left[\hat{a}_{\tilde{n}+1}, \hat{a}_{1}\right]\right\}$ for any $h^{T+1} \in H^{T+1}$. Therefore, 
at $h^{T+1}$ in which $I\left(h^{T+1}\right)=\left[\hat{a}_{i+1}, \hat{a}_{i}\right]$ for some $i \in\{0, \ldots \tilde{n}\}$, the optimal project for $R$ is $\bar{y}\left(\hat{a}_{i+1}, \hat{a}_{i}\right) \equiv \arg \max _{y} \int_{\hat{a}_{i+1}}^{\hat{a}_{i}} u^{R}(y, \theta) g(\theta) d \theta$, and at $h^{T+1}$ in which $I\left(h^{T+1}\right)=\left[\hat{a}_{\tilde{n}+1}, \hat{a}_{1}\right]$, the optimal project for $R$ is $\bar{y}\left(\hat{a}_{\tilde{n}+1}, \hat{a}_{1}\right) \equiv \arg \max _{y} \int_{\hat{a}_{0}}^{\hat{a}_{1}} u^{R}(y, \theta) g(\theta) d \theta$. Hence, $\hat{\rho}\left(h^{T+1}\right)$ becomes $\bar{y}\left(\hat{a}_{i+1}, \hat{a}_{i}\right)$ at $h^{T+1}$ in which $I\left(h^{T+1}\right)=\left[\hat{a}_{i+1}, \hat{a}_{i}\right]$ for some $i \in\{0, \ldots \tilde{n}\}$, and $\hat{\rho}\left(h^{T+1}\right)$ becomes $\bar{y}\left(\hat{a}_{\tilde{n}+1}, \hat{a}_{1}\right)$ at $h^{T+1}$ in which $I\left(h^{T+1}\right)=\left[\hat{a}_{\tilde{n}+1}, \hat{a}_{1}\right]$. It is obvious that $\hat{\rho}\left(h^{T+1}\right)$ is an optimal action for $R$ at any $h^{T+1} \in H^{T+1}$.

In period $t \geq 2, R$ always pays nothing to $S$. This implies that $\left\{\left[\hat{a}_{i+1}, \hat{a}_{i}\right)\right\}_{i=1}^{\tilde{n}}$ must coincide with the $\tilde{n}$-element equilibrium partition achieved in a model with one shot information transmission where $\theta$ is drawn from a distribution with density $\left\{g(\theta) / \int_{0}^{\hat{a}_{1}} g(\theta) d \theta\right\} \cdot \mathbb{1}_{\left[0, \hat{a}_{1}\right)}(\theta)$. Therefore, the boundaries of this partition, $\left\{\left[\hat{a}_{i+1}, \hat{a}_{i}\right)\right\}_{i=1}^{\tilde{n}}$, must be solutions to the following a nonlinear difference equation whose initial and terminal conditions are $a_{1}=\hat{a}_{1}$ and $a_{\tilde{n}+1}=0$, respectively: for $i=2, \ldots, \tilde{n}$,

$$
s \cdot u^{S}\left(\bar{y}\left(a_{i+1}, a_{i}\right), a_{i}, b\right)-s \cdot u^{S}\left(\bar{y}\left(a_{i}, a_{i-1}\right), a_{i}, b\right)=0 .
$$

When $\hat{a}_{1}=1$, the solution to (25) induces a partition which coincides with $\tilde{n}$-element equilibrium partition in a model with one shot information transmission. Moreover, the solution to (25), vary continuously with respect to initial condition $a_{1}=\hat{a}_{1}$, and we now suppose that the solution to (1)-(3), $\left\{\tilde{a}_{0}, \ldots, \tilde{a}_{\tilde{n}}\right\}$, induces a partition: $0=\tilde{a}_{\tilde{n}}<\cdots<\tilde{a}_{0}=1$. Therefore, there exists $\underline{x}<1$ such that (25) is well-defined for all $\hat{a}_{1} \in(\underline{x}, 1)$. Let $\underline{a}_{1}$ be the minimum value of $\underline{x}$ such that for all $\hat{a}_{1} \in(\underline{x}, 1)$, the solution to (25) induces a $\tilde{n}$-element partition: $0=a_{\tilde{n}+1}<a_{\tilde{n}}<\cdots<a_{1}=\hat{a}_{1}$. Since the solution to (25) does not depends on both $s$ and $r$, the value of $\underline{a}_{1}$ also does not depends on both $s$ and $r$.

If $\left\{\hat{a}_{2}, \ldots, \hat{a}_{\tilde{n}+1}\right\}$ is a solution to $(25)$ where $\hat{a}_{1} \in\left(\underline{a}_{1}, 1\right)$, there is no profitable deviation for $S$ from $\hat{\sigma}$ at any $h_{\theta}^{(2,1)}$ in which $m_{1}<\hat{a}_{1}$ and $w_{1} \geq w^{*}$. Moreover, $S$ always sends a babbling message at any $h_{\theta}^{(2,1)}$ in which $m_{1} \geq \hat{a}_{1}$, or $m_{1}<a_{1}$ and $w_{1}<w^{*}$. The same can be said at any $h_{\theta}^{(t, 1)}$ for $t \geq 3$. This implies that if $\left\{\hat{a}_{1}, \ldots, \hat{a}_{\tilde{n}+1}\right\}$ is a solution to (25) where $\hat{a}_{1} \in\left(\underline{a}_{1}, 1\right)$, then $\hat{\sigma}$ is optimal for $S$ at any $h_{\theta}^{(t, 1)}$ for $t \geq 2$. Therefore, in what follows, we suppose that $\left\{\hat{a}_{2}, \ldots, \hat{a}_{\tilde{n}+1}\right\}$ is a solution to $(25)$ where $\hat{a}_{1} \in\left(\underline{a}_{1}, 1\right)$.

At stage 1 in period 1 , if $S$ of type $\theta$ sends $m_{1} \geq \hat{a}_{1}$, then he obtains $s \cdot u^{S}\left(\bar{y}\left(\hat{a}_{1}, 1\right), \theta, b\right)$. Otherwise, $S$ of type $\theta \geq \hat{a}_{2}$ obtains $s \cdot u^{S}\left(\bar{y}\left(\hat{a}_{2}, \hat{a}_{1}\right), \theta, b\right)+w^{*}$, and $S$ of type $\theta \in\left[\hat{a}_{i+1}, \hat{a}_{i}\right)$, for $i \geq 2$, obtains $s \cdot u^{S}\left(\bar{y}\left(\hat{a}_{i+1}, \hat{a}_{i}\right), \theta, b\right)+w^{*}$. We assume that $u_{11}^{S}<0$ and $u_{12}^{S}>0$. Moreover, it holds that $\bar{y}\left(\hat{a}_{i+1}, \hat{a}_{i}\right)>\bar{y}\left(\hat{a}_{i}, \hat{a}_{i-1}\right)$. Therefore, if it is satisfied that

$$
\begin{aligned}
& s \cdot u^{S}\left(\bar{y}\left(\hat{a}_{1}, 1\right), \hat{a}_{1}, b\right)-s \cdot u^{S}\left(\bar{y}\left(\hat{a}_{2}, \hat{a}_{1}\right), \hat{a}_{1}, b\right)=w^{*}, \quad \text { then } \\
& s \cdot u^{S}\left(\bar{y}\left(\hat{a}_{1}, 1\right), \theta, b\right) \geq \max _{j \in\{1, \ldots, \tilde{n}\}} s \cdot u^{S}\left(\bar{y}\left(\hat{a}_{j+1}, \hat{a}_{j}\right), \theta, b\right)+w^{*} \text { for } \theta \geq \hat{a}_{1}, \quad \text { and } \\
& s \cdot u^{S}\left(\bar{y}\left(\hat{a}_{i+1}, \hat{a}_{i}\right), \theta, b\right)+w^{*}>s \cdot u^{S}\left(\bar{y}\left(\hat{a}_{1}, 1\right), \theta, b\right) \quad \text { for } i \geq 1 \text { and } \theta \in\left[\hat{a}_{i+1}, \hat{a}_{i}\right) .
\end{aligned}
$$

When (27) and (28) hold, $S$ has no incentive to deviate from $\hat{\sigma}$ at stage 1 in period 1. Since we assume that $R^{\prime}$ s payment must be nonnegative, $w^{*}$ must be nonnegative. We now make sure that $R^{\prime}$ s payment, $w^{*}\left(\hat{a}_{1}\right) \equiv s \cdot u^{S}\left(\bar{y}\left(\hat{a}_{1}, 1\right), \hat{a}_{1}, b\right)-s \cdot u^{S}\left(\bar{y}\left(\hat{a}_{2}, \hat{a}_{1}\right), \hat{a}_{1}, b\right)$, that holds the equation (26) is positive for any $\hat{a}_{1} \in\left(\underline{a}_{1}, 1\right)$. If $w^{*}\left(\hat{a}_{1}\right)=0$ for some $\hat{a}_{1} \in\left(\underline{a}_{1}, 1\right)$, then (1)-(3) has a solutions: $0=\hat{a}_{\tilde{n}+1}<\hat{a}_{\tilde{n}}<\cdots<\hat{a}_{0}=1$. This is incompatible 
with the definition of $\tilde{n}$. Since $\bar{y}\left(\hat{a}_{1}, 1\right), \bar{y}\left(\hat{a}_{2}, \hat{a}_{1}\right)$ and $\hat{a}_{2}$ is continuous in $\hat{a}_{1} \in\left(\underline{a}_{1}, 1\right]$, $w^{*}\left(\hat{a}_{1}\right)$ is continuous in $\hat{a}_{1} \in\left(\underline{a}_{1}, 1\right]$. Since, moreover, $\hat{a}_{2}=\tilde{a}_{1}$ when $\hat{a}_{1}=1$, we have $w^{*}(1)=s \cdot u^{S}\left(y^{R}(1), 1, b\right)-s \cdot u^{S}\left(\bar{y}\left(\tilde{a}_{1}, 1\right), 1, b\right)$. Note that $w^{*}(1)>0$ since $u_{11}^{S}<0$ and $\bar{y}\left(\tilde{a}_{1}, 1\right)<y^{R}(1)<y^{S}(1, b)$. Therefore, $w^{*}\left(\hat{a}_{1}\right)>0$ for any $\hat{a}_{1} \in\left(\underline{a}_{1}, 1\right]$.

At any $h^{(t, 2)}, R$ has no incentive to increase the amount of payment because that does not affect $S$ 's behavior. Therefore, we have only to make sure the optimality of $\rho$ at $h^{(1,2)}$ in which $m_{1}<\hat{a}_{1}$. At history $h^{(1,2)}$ in which $m_{1}<\hat{a}_{1}$, if $R$ pays $w_{1}<w^{*}$, then she obtains $\bar{u}\left(w_{1}\right)$ :

$$
\bar{u}\left(w_{1}\right)=-w_{1}+\frac{r}{G\left(\hat{a}_{1}\right)} \int_{0}^{\hat{a}_{1}} u^{R}\left(\bar{y}\left(0, \hat{a}_{1}\right), \theta\right) g(\theta) d \theta .
$$

Clearly, $\bar{u}\left(w_{1}\right)$ takes the maximum value $\bar{u}$ at $w_{1}=0$. On the other hand, by paying $w_{1} \geq w^{*}$ at history $h^{(1,2)}$ in which $m_{1}<a_{1}$, she obtains $u^{*}\left(w_{1}\right)$ :

$$
u^{*}\left(w_{1}\right)=-w_{1}+\frac{r}{G\left(\hat{a}_{1}\right)} \sum_{i=1}^{\tilde{n}} \int_{\hat{a}_{i+1}}^{\hat{a}_{i}} u^{R}\left(\bar{y}\left(\hat{a}_{i+1}, \hat{a}_{i}\right), \theta\right) g(\theta) d \theta .
$$

Clearly, $u^{*}\left(w_{1}\right)$ takes the maximum value $u^{*}$ at $w_{1}=w^{*}$. Therefore, paying $w^{*}$ is an optimal decision for $R$ at $h^{(1,2)}$ in which $m_{1}<\hat{a}_{1}$ if and only if

$$
\begin{aligned}
& u^{*} \geq \bar{u} \Longleftrightarrow \\
& \frac{r}{G\left(\hat{a}_{1}\right)} \sum_{i=1}^{\tilde{n}} \int_{\hat{a}_{i+1}}^{\hat{a}_{i}} u^{R}\left(\bar{y}\left(\hat{a}_{i+1}, \hat{a}_{i}\right), \theta\right) g(\theta) d \theta-\frac{r}{G\left(\hat{a}_{1}\right)} \int_{0}^{\hat{a}_{1}} u^{R}\left(\bar{y}\left(0, \hat{a}_{1}\right), \theta\right) g(\theta) d \theta \geq w^{*}(29)
\end{aligned}
$$

We denote by $r \cdot V\left(\hat{a}_{1}\right)$ the left-hand side of the inequality (29). $V\left(\hat{a}_{1}\right)$ is continuous in $\hat{a}_{1} \in\left(\underline{a}_{1}, 1\right]$, and $V\left(\hat{a}_{1}\right)>0$ for $\hat{a}_{1} \in\left(\underline{a}_{1}, 1\right]$. Moreover, $V(1)=E U_{C S, \tilde{n}}^{R}-E U_{C S, u i}^{R}$ where $E U_{C S, \tilde{n}}^{R} \equiv \sum_{i=1}^{\tilde{n}} \int_{\tilde{a}_{i}}^{\tilde{a}_{i-1}} u^{R}\left(\bar{y}\left(\tilde{a}_{i}, \tilde{a}_{i-1}\right), \theta\right) g(\theta) d \theta$ and $E U_{C S, u i}^{R} \equiv \int_{0}^{1} u^{R}(\bar{y}(0,1), \theta) g(\theta) d \theta$. Let $\alpha\left(\hat{a}_{1}\right)$ be $u^{S}\left(\bar{y}\left(\hat{a}_{1}, 1\right), \hat{a}_{1}, b\right)-u^{S}\left(\bar{y}\left(\hat{a}_{2}, \hat{a}_{1}\right), \hat{a}_{1}, b\right)$. In what follows, $s \cdot \alpha\left(\hat{a}_{1}\right)$ denotes $R$ 's payment, $w^{*}\left(\hat{a}_{1}\right)$, that holds the equation (26). The inequality (29) can be simplified into $\frac{s}{r} \leq \frac{V\left(\hat{a}_{1}\right)}{\alpha\left(\hat{a}_{1}\right)}$. It is obvious that $\frac{V\left(\hat{a}_{1}\right)}{\alpha\left(\hat{a}_{1}\right)}$ is continuous in $\hat{a}_{1} \in\left(\underline{a}_{1}, 1\right]$, and

$$
\frac{V(1)}{\alpha(1)}=\frac{E U_{C S, \tilde{n}}^{R}-E U_{C S, u i}^{R}}{u^{S}\left(y^{*}(1), 1, b\right)-u^{S}\left(\bar{y}\left(\tilde{a}_{1}, 1\right), 1, b\right)}>0 .
$$

Therefore, if $\frac{s}{r}<\eta(b) \equiv \frac{V(1)}{\alpha(1)}$, then $\left\{\hat{a}_{1} \in\left(\underline{a}_{1}, 1\right): s / r \leq V\left(\hat{a}_{1}\right) / \alpha\left(\hat{a}_{1}\right)\right\} \neq \emptyset$. This implies that if $\frac{s}{r}<\eta(b)$, there exists a nonempty set $\left\{\hat{a}_{1} \in\left(\underline{a}_{1}, 1\right): s / r \leq V\left(\hat{a}_{1}\right) / \alpha\left(\hat{a}_{1}\right)\right\}$ such that $((\hat{\sigma}, \hat{\rho}), \hat{f})$ constitutes a $(\tilde{n}+1)$-element partition equilibrium when $\hat{a}_{1} \in\left\{\hat{a}_{1} \in\left(\underline{a}_{1}, 1\right)\right.$ : $\left.s / r \leq V\left(\hat{a}_{1}\right) / \alpha\left(\hat{a}_{1}\right)\right\} . \diamond$

Remark 4. Since $\frac{V(1)}{\alpha(1)}=\eta(b)>0$ and $\frac{V\left(\hat{a}_{1}\right)}{\alpha\left(\hat{a}_{1}\right)}$ is continuous in $\hat{a}_{1} \in\left(\underline{a}_{1}, 1\right]$, there exists $z \in\left(\underline{a}_{1}, 1\right)$ such that $\frac{s}{r} \leq \frac{V\left(\hat{a}_{1}\right)}{\alpha\left(\hat{a}_{1}\right)}$ holds for any $\hat{a}_{1} \in(z, 1)$. Let $\underline{a}_{1}(s / r)$ be the infimum value of $z$. Since $\frac{V\left(\hat{a}_{1}\right)}{\alpha\left(\hat{a}_{1}\right)}$ is continuous in $\hat{a}_{1} \in\left(\underline{a}_{1}, 1\right]$ and $\frac{V\left(\hat{a}_{1}\right)}{\alpha\left(\hat{a}_{1}\right)}>0$ for all $\hat{a}_{1} \in\left(\underline{a}_{1}, 1\right]$, the value of $\underline{a}_{1}(s / r)$ is strictly decreasing and goes to $\underline{a}_{1}$ as $\frac{s}{r}$ goes to 0 . 


\section{Appendix H Proof of Lemma 2}

Crawford and Sobel have shown that in a model with one-shot information transmission, under Condition $M, S$ always strictly prefers ex ante $\tilde{n}$-element partition equilibrium to any other equilibria. We have

$$
E U_{C S}^{S}=s \sum_{i=1}^{\tilde{n}} \int_{\tilde{a}_{i}}^{\tilde{a}_{i-1}} u^{S}\left(\bar{y}\left(\tilde{a}_{i}, \tilde{a}_{i-1}\right), \theta, b\right) g(\theta) d \theta .
$$

By Proposition 1, it must be satisfied that $\frac{s}{r}<\eta(b)$ in order for an equilibrium $((\hat{\sigma}, \hat{\rho}), \hat{f})$ to exist. Therefore, in what follows, we suppose that $\frac{s}{r}<\eta(b)$.

The ex ante expected payoff of $S$ under $((\hat{\sigma}, \hat{\rho}), \hat{f})$ is

$$
E \hat{U}^{S}(x)=s\left[\sum_{i=1}^{\tilde{n}+1} \int_{\hat{a}_{i}^{x}}^{\hat{a}_{i-1}^{x}} u^{S}\left(\bar{y}\left(\hat{a}_{i}^{x}, \hat{a}_{i-1}^{x}\right), \theta, b\right) g(\theta) d \theta+G(x) \cdot \alpha(x)\right] .
$$

Recall that $s \cdot \alpha(x) \equiv w^{*}(x)=s \cdot u^{S}(\bar{y}(x, 1), x, b)-s \cdot u^{S}\left(\bar{y}\left(\hat{a}_{2}^{x}, x\right), x, b\right)$ is positive for $x>\underline{a}_{1}$, and $s \cdot \alpha(x)$ is continuous in $x>\underline{a}_{1}$.

Let $\Delta(x)$ denote $E \hat{U}^{S}(x)-E U_{C S}^{S}$. Since $\lim _{x \uparrow 1} \Delta(x)=\alpha(1)>0$ and $\Delta(x)$ is continuous in $x \in\left(\underline{a}_{1}, 1\right]$, there exists $d<1$ such that $d \geq \underline{a}_{1}(s / r)$ and

$$
\Delta(x)>0 \text { for all } x \in(d, 1) .
$$

This completes the proof of Lemma 2. $\diamond$

Remark 5. Define $\underline{d}(s / r) \equiv \inf \left\{d: d \geq \underline{a}_{1}(s / r)\right.$ and $\Delta(x)>0$ for all $\left.x \in(d, 1)\right\}$. Since $\underline{a}_{1}(s / r)$ is decreasing as $\frac{s}{r}$ is decreasing and $\Delta(x)$ does not depend on both $s$ and $r$, it is satisfied that $\underline{d}(s / r)$ is decreasing (but not always strictly decreasing) as $\frac{s}{r}$ is decreasing.

\section{Appendix I Proof of Lemma 3}

In common with the proof of Lemma 2, we suppose that $\frac{s}{r}<\eta(b)$.

Let $\delta(x, s, r)$ denote $\left\{E \hat{U}^{R}(x)-E U_{C S}^{R}\right\} / r$. We have

$$
\delta(x, s, r)=\hat{W}(x)-\frac{s}{r} G(x) \cdot \alpha(x)-\sum_{i=1}^{\tilde{n}} \int_{\tilde{a}_{i}}^{\tilde{a}_{i-1}} u^{R}\left(\bar{y}\left(\tilde{a}_{i}, \tilde{a}_{i-1}\right), \theta\right) g(\theta) d \theta .
$$

It holds that $\delta(x, s, r)<0$, if and only if

$$
\bar{\eta}(b, x) \equiv \frac{\hat{W}(x)-\sum_{i=1}^{\tilde{n}} \int_{\tilde{a}_{i}}^{\tilde{a}_{i-1}} u^{R}\left(\bar{y}\left(\tilde{a}_{i}, \tilde{a}_{i-1}\right), \theta\right) g(\theta) d \theta}{G(x) \cdot \alpha(x)}<\frac{s}{r} .
$$

Since $\bar{\eta}(b, x)$ is continuous in $x \in\left(\underline{a}_{1}, 1\right]$ and $\bar{\eta}(b, 1)=0$, there exists $\hat{\varepsilon}>0$ such that $\bar{\eta}(b, \underline{x})<\frac{s}{r}$ for all $\underline{x} \in(1-\hat{\varepsilon}, 1)$.

It holds that $\delta(x, s, r)>0$, if and only if

$$
\bar{\eta}(b, x)>\frac{s}{r} .
$$


Since $x$ belongs to $\left(\underline{a}_{1}(s / r), 1\right)$ and $\inf _{x \in\left(\underline{a}_{1}(s / r), 1\right)} G(x) \alpha(x)>0, \bar{\eta}(b, x)$ has a least upper bound $\bar{\eta}(b \mid s / r)=\sup _{x \in\left(\underline{a}_{1}(s / r), 1\right)} \bar{\eta}(b, x)$. Under Condition $C, \bar{\eta}(b, x)>0$ for some $x \in$ $\left(\underline{a}_{1}(s / r), 1\right)$. This implies that $\bar{\eta}(b \mid s / r)>0$. Since, moreover, $\underline{a}_{1}(s / r)$ is not increasing as $\frac{s}{r}$ is decreasing, $\bar{\eta}(b \mid s / r)$ is not decreasing as $\frac{s}{r}$ is decreasing. Therefore, we can take a supremum of the value of $\frac{s}{r}$ that satisfies $\bar{\eta}(b \mid s / r)>\frac{s}{r}$. We denote by $\bar{\eta}(b)$ this supremum. Note that $\bar{\eta}(b)<+\infty$ since $\hat{W}(x)-\sum_{i=1}^{\tilde{n}} \int_{\tilde{a}_{i}}^{\tilde{a}_{i-1}} u^{R}\left(\bar{y}\left(\tilde{a}_{i}, \tilde{a}_{i-1}\right), \theta\right) g(\theta) d \theta<+\infty$ for any $x \in\left(\underline{a}_{1}, 1\right)$. This completes the proof of Lemma 3 . $\diamond$

Remark 6. When $x$ almost equal to 1 , the partition under the $(\tilde{n}+1)$-element partition equilibrium almost coincides with the partition under the $\tilde{n}$-element partition equilibrium in a model with one-shot information transmission. Nevertheless, the expected payment of monetary transfer is high (almost coincides with $s \alpha(1)$ ). Therefore, if $\frac{s}{r}<\eta(b)$, there always exists a $(\tilde{n}+1)$-element partition equilibrium which is unfavorable to $R$.

\section{Appendix J Proof of Proposition 13}

In common with the proof of Lemma 2, we suppose that $\frac{s}{r}<\eta(b)$.

Let $\delta(x, s, r)$ denote $\left\{E \hat{U}^{R}(x)-E U_{C S}^{R}\right\} / r$. We have

$$
\delta(x, s, r)=\hat{W}(x)-\frac{s}{r} G(x) \cdot \alpha(x)-\sum_{i=1}^{\tilde{n}} \int_{\tilde{a}_{i}}^{\tilde{a}_{i-1}} u^{R}\left(\bar{y}\left(\tilde{a}_{i}, \tilde{a}_{i-1}\right), \theta\right) g(\theta) d \theta .
$$

It holds that $\delta(x, s, r)<0$, if and only if

$$
\bar{\eta}(b, x) \equiv \frac{\hat{W}(x)-\sum_{i=1}^{\tilde{n}} \int_{\tilde{a}_{i}}^{\tilde{a}_{i-1}} u^{R}\left(\bar{y}\left(\tilde{a}_{i}, \tilde{a}_{i-1}\right), \theta\right) g(\theta) d \theta}{G(x) \cdot \alpha(x)}<\frac{s}{r} .
$$

Since $\bar{\eta}(b, x)$ is continuous in $x \in\left(\underline{a}_{1}, 1\right]$ and $\bar{\eta}(b, 1)=0$, there exists $\hat{\varepsilon}>0$ such that $\bar{\eta}(b, \underline{x})<\frac{s}{r}$ for all $\underline{x} \in(1-\hat{\varepsilon}, 1)$.

It holds that $\delta(x, s, r)>0$, if and only if

$$
\bar{\eta}(b, x)>\frac{s}{r} .
$$

Since $x$ belongs to $\left(\underline{a}_{1}(s / r), 1\right)$ and $\inf _{x \in\left(\underline{a}_{1}(s / r), 1\right)} G(x) \alpha(x)>0, \bar{\eta}(b, x)$ has a least upper bound $\bar{\eta}(b \mid s / r)=\sup _{x \in\left(a_{1}(s / r), 1\right)} \bar{\eta}(b, x)$. Under Condition $C, \bar{\eta}(b, x)>0$ for some $x \in$ $\left(\underline{a}_{1}(s / r), 1\right)$. This implies that $\bar{\eta}(b \mid s / r)>0$. Since, moreover, $\underline{a}_{1}(s / r)$ is not increasing as $\frac{s}{r}$ is decreasing, $\bar{\eta}(b \mid s / r)$ is not decreasing as $\frac{s}{r}$ is decreasing. Therefore, we can take a supremum of the value of $\frac{s}{r}$ that satisfies $\bar{\eta}(b \mid s / r)>\frac{s}{r}$. We denote by $\bar{\eta}(b)$ this supremum. Note that $\bar{\eta}(b)<+\infty$ since $\hat{W}(x)-\sum_{i=1}^{\tilde{n}} \int_{\tilde{a}_{i}}^{\tilde{a}_{i-1}} u^{R}\left(\bar{y}\left(\tilde{a}_{i}, \tilde{a}_{i-1}\right), \theta\right) g(\theta) d \theta<+\infty$ for any $x \in\left(a_{1}, 1\right)$. This completes the proof of Lemma 3 . $\diamond$

Remark 7. When $x$ almost equal to 1 , the partition under the $(\tilde{n}+1)$-element partition equilibrium almost coincides with the partition under the $\tilde{n}$-element partition equilibrium in a model with one-shot information transmission. Nevertheless, the expected payment of monetary transfer is high (almost coincides with $s \alpha(1)$ ). Therefore, if $\frac{s}{r}<\eta(b)$, there always exists a $(\tilde{n}+1)$-element partition equilibrium which is unfavorable to $R$. 


\section{Appendix K Condition C}

Suppose that $s \cdot u^{S}(y, \theta, b) \equiv-s(y-(\theta+b))^{2}, r \cdot u^{R}(y, \theta) \equiv-r(y-\theta)^{2}$, and $G(\theta)$ is uniform distribution over $[0,1]$. In this case, the the boundaries of the partition induced from $((\hat{\sigma}, \hat{\rho}), \hat{f})$ are given by

$$
\hat{a}_{i}^{x}= \begin{cases}1 & \text { for } i=0, \\ x & \text { for } i=1, \\ \frac{\tilde{n}+1-i}{\tilde{n}} x-2 b(\tilde{n}+1-i)(i-1) & \text { for } i=2, \ldots, \tilde{n} \\ 0 & \text { for } i=\tilde{n}+1 .\end{cases}
$$

We have already shown that for $\tilde{n} \geq 2$, there exists $\eta(b)$ such that if $\frac{s}{r}<\eta(b)$, then $((\hat{\sigma}, \hat{\rho}), \hat{f})$ constitutes an equilibrium whose partition induced by $\hat{a}_{i}^{x}$ where $x \in\left(\underline{a}_{1}(s / r), 1\right)$. Note that $\bar{y}\left(\hat{a}_{i+1}^{x}, \hat{a}_{i}^{x}\right)=\frac{\hat{a}_{i+1}^{x}+\hat{a}_{i}^{x}}{2}$ for $i=0, \ldots, \tilde{n}$.

The Envelope Theorem yields

$$
\frac{d}{d x} \hat{W}(x)=\sum_{i=1}^{\tilde{n}} g\left(\hat{a}_{i}^{x}\right) \frac{d \hat{a}_{i}^{x}}{d x}\left[u^{R}\left(\bar{y}\left(\hat{a}_{i+1}^{x}, \hat{a}_{i}^{x}\right), \hat{a}_{i}^{x}\right)-u^{R}\left(\bar{y}\left(\hat{a}_{i}^{x}, \hat{a}_{i-1}^{x}\right), \hat{a}_{i}^{x}\right)\right] .
$$

Since $\lim _{x \uparrow 1} \hat{a}_{i}^{x}=\tilde{a}_{i-1}$, we have

$$
\begin{aligned}
\left.\frac{d}{d x}\right|_{x=1} \hat{W}(x)= & \left.\sum_{j=1}^{\tilde{n}-1} g\left(\tilde{a}_{j}\right)\left[u^{R}\left(\bar{y}\left(\tilde{a}_{j+1}, \tilde{a}_{j}\right), \tilde{a}_{j}\right)-u^{R}\left(\bar{y}\left(\tilde{a}_{j}, \tilde{a}_{j-1}\right), \tilde{a}_{j}\right)\right] \frac{d}{d x}\right|_{x=1} \hat{a}_{j+1}^{x} \\
& +\left.g\left(\tilde{a}_{0}\right)\left[u^{R}\left(\bar{y}\left(\tilde{a}_{1}, \tilde{a}_{0}\right), \tilde{a}_{0}\right)-u^{R}\left(y^{*}\left(\tilde{a}_{0}\right), \tilde{a}_{0}\right)\right] \frac{d}{d x}\right|_{x=1} \hat{a}_{1}^{x} .
\end{aligned}
$$

Therefore, we have

$$
\left.\frac{d}{d x}\right|_{x=1} \hat{W}(x)=\sum_{j=1}^{\tilde{n}-1}\left[-\left(\frac{\tilde{a}_{j+1}-\tilde{a}_{j}}{2}\right)^{2}+\left(\frac{-\tilde{a}_{j}+\tilde{a}_{j-1}}{2}\right)^{2}\right] \frac{\tilde{n}-j}{\tilde{n}}-\left(\frac{1-\tilde{a}_{1}}{2}\right)^{2} .
$$

Since $\tilde{a}_{j}=\frac{\tilde{n}-j}{\tilde{n}}-2 b j(\tilde{n}-j)$,

$$
-\left(\frac{\tilde{a}_{j+1}-\tilde{a}_{j}}{2}\right)^{2}+\left(\frac{-\tilde{a}_{j}+\tilde{a}_{j-1}}{2}\right)^{2}>0 \text { for } j=1, \ldots, \tilde{n}-1 .
$$

Moreover,

$$
\begin{aligned}
\sum_{j=1}^{\tilde{n}-1}\left[-\left(\frac{\tilde{a}_{j+1}-\tilde{a}_{j}}{2}\right)^{2}+\left(\frac{-\tilde{a}_{j}+\tilde{a}_{j-1}}{2}\right)^{2}\right] \frac{\tilde{n}-j}{\tilde{n}} & <\sum_{j=1}^{\tilde{n}-1}\left[-\left(\frac{\tilde{a}_{j+1}-\tilde{a}_{j}}{2}\right)^{2}+\left(\frac{-\tilde{a}_{j}+\tilde{a}_{j-1}}{2}\right)^{2}\right] \\
& <\left(\frac{1-\tilde{a}_{1}}{2}\right)^{2} .
\end{aligned}
$$

This establishes $\left.\frac{d}{d x}\right|_{x=1} \hat{W}(x)<0$. 
Remark 8. Suppose that $s \cdot u^{S}(y, \theta, b) \equiv-s(y-(\theta+b))^{2}, r \cdot u^{R}(y, \theta) \equiv-r(y-\theta)^{2}$, and $G(\theta)$ is a distribution over $[0,1]$ with a density $g(\theta)=-2 \theta+2$. By the Theorem 2 in Crawford and Sobel (1982), all solution to (1) satisfy Condition M. By Condition M and $u_{13}^{S}>0$, we have $d \hat{a}_{i}^{x} / d x>0$ and

$$
u^{R}\left(\bar{y}\left(\tilde{a}_{j+1}, \tilde{a}_{j}\right), \tilde{a}_{j}\right)-u^{R}\left(\bar{y}\left(\tilde{a}_{j}, \tilde{a}_{j-1}\right), \tilde{a}_{j}\right) \geq u^{S}\left(\bar{y}\left(\tilde{a}_{j+1}, \tilde{a}_{j}\right), \tilde{a}_{j}, b\right)-u^{S}\left(\bar{y}\left(\tilde{a}_{j}, \tilde{a}_{j-1}\right), \tilde{a}_{j}, b\right) \geq 0 .
$$

Since $g(1)=0$, this means that $\left.\frac{d}{d x}\right|_{x=1} \hat{W}(x)>0$.

\section{Appendix L Construction of $\mathbb{P}$}

Let $\sigma^{1}: \mathbb{B}\left(M_{1}\right) \times \Theta \rightarrow[0,1]$ and $\mathbb{G}: \mathbb{B}(\Theta) \rightarrow[0,1]$ be $S^{\prime}$ s behavior strategy at stage 1 in period 1 and the prior probability measure on $(\Theta, \mathbb{B}(\Theta))$, respectively. Note that $\sigma^{1}(\tilde{M}, \cdot): \Theta \rightarrow[0,1]$ is measurable for each $\tilde{M} \in \mathbb{B}\left(M_{1}\right)$. First, we induce a product measure $\mathbb{P}_{\sigma}^{1}$ on $\left(\Theta \times M_{1}, \mathbb{B}(\Theta) \otimes \mathbb{B}\left(M_{1}\right)\right)$ as follows: for each $\tilde{\Theta} \in \mathbb{B}(\Theta)$ and $\tilde{M} \in \mathbb{B}\left(M_{1}\right)$,

$$
\mathbb{P}_{\sigma}^{1}(\tilde{\Theta} \times \tilde{M})=\int_{\theta \in \tilde{\Theta}} \sigma^{1}(\tilde{M}, \theta) \mathrm{G}(d \theta) .
$$

Let $\rho_{1}^{-1}(\tilde{W})$ be the set of $m_{1}$ such that $\left\{m_{1} \in M_{1}: \rho\left(m_{1}\right) \in \tilde{W}\right.$ for $\tilde{W} \in \mathbb{B}\left(W_{1}\right)$. Since we assume that $\rho: M_{1} \rightarrow W_{1}$ is a measurable, it is satisfied that $\rho_{1}^{-1}(\tilde{W}) \in \mathbb{B}\left(M_{1}\right)$ We induce a product measure $\mathbb{P}_{\sigma, \rho}^{1}$ on $\left(\Theta \times M_{1} \times W_{1}, \mathbb{B}(\Theta) \otimes \mathbb{B}\left(M_{1}\right) \otimes \mathbb{B}\left(W_{1}\right)\right)$ as: for each $\tilde{\Theta} \in \mathbb{B}(\Theta)$, $\tilde{M} \in \mathbb{B}\left(M_{1}\right)$ and $\tilde{W} \in \mathbb{B}\left(W_{1}\right)$,

$$
\mathbb{P}_{\sigma, \rho}^{1}(\tilde{\Theta} \times \tilde{M} \times \tilde{W})=\mathbb{P}_{\sigma}^{1}\left(\tilde{\Theta} \times\left\{\tilde{M} \cap \rho_{1}^{-1}(\tilde{W})\right\}\right),
$$

Next, we induce a product measure $\mathbb{P}_{\sigma}^{2}$ on $\left(H_{\Theta}^{(2,1)} \times M_{2}, \mathbb{B}\left(H_{\Theta}^{(2,1)}\right) \otimes \mathbb{B}\left(M_{1}\right)\right)$ as follows: for each $\tilde{H} \in \mathbb{B}\left(H_{\Theta}^{(2,1)}\right)$ and $\tilde{M} \in \mathbb{B}\left(M_{2}\right)$,

$$
\mathbb{P}_{\sigma}^{2}(\tilde{H} \times \tilde{M})=\int_{h \in \tilde{H}} \sigma^{2}(\tilde{M}, h) \mathbb{P}_{\sigma, \rho}^{1}(d h)
$$

where $\sigma^{2}: \mathbb{B}\left(M_{2}\right) \times H_{\Theta}^{(2,1)} \rightarrow[0,1]$ denotes $S^{\prime}$ s behavior strategy at stage 1 in period 2 . By the repeated application of the above result, we can obtain $\mathbb{P}$. Under the given strategy profile and belief system $((\sigma, \rho), f)$, in the same way, we can uniquely determine a probability measure over the outcomes after any (public or private) history.

\section{Appendix M Consistency of the belief system}

Given $h^{(t, 2)}$, the belief system induces a probability measure $f\left(\cdot \mid h^{(t, 2)}\right)$ on $(\Theta, \mathbb{B}(\Theta))$. Since, moreover, the $S^{\prime}$ s behavior strategy $\sigma\left(\tilde{M}, \cdot \mid h^{(t, 2)}, w_{t}\right): \Theta \rightarrow[0,1]$ is measurable for any $\tilde{M} \in \mathbb{B}\left(M_{t+1}\right)$ and $w_{t} \in W_{t}$. we can uniquely define a probability measure $\mathbb{P}\left(\cdot \mid h^{(t, 2)}, w_{t}\right)$ on $\left(\Theta \times M_{t+1}, \mathbb{B}(\Theta) \otimes \mathbb{B}\left(M_{t+1}\right)\right)$ as follows: for $\tilde{\Theta} \in \mathbb{B}(\Theta)$ and $\tilde{M} \in \mathbb{B}\left(M_{t+1}\right)$,

$$
\mathbb{P}\left(\tilde{\Theta} \times \tilde{M} \mid h^{(t, 2)}, w_{t}\right) \equiv \int_{\tilde{\Theta}} \sigma\left(\tilde{M}, \theta \mid h^{(t, 2)}, w_{t}\right) f\left(d \theta \mid h^{(t, 2)}\right) .
$$


Therefore, we calculate the posterior belief: if $\mathbb{P}\left(\Theta \times \tilde{M} \mid h^{(t, 2)}, w_{t}\right)>0$, then

$$
f\left(\tilde{\Theta} \mid h^{(t, 2)}, w_{t}, \tilde{M}\right)=\frac{\mathbb{P}\left(\tilde{\Theta} \times \tilde{M} \mid h^{(t, 2)}, w_{t}\right)}{\mathbb{P}\left(\Theta \times \tilde{M} \mid h^{(t, 2)}, w_{t}\right)} .
$$

Moreover, fix $\tilde{\Theta} \in \mathbb{B}(\Theta)$, then $\mathbb{P}\left(\tilde{\Theta}, \mid \cdot h^{(t, 2)}, w_{t}\right)$ and $\mathbb{P}\left(\Theta, \cdot \mid h^{(t, 2)}, w_{t}\right)$ induce measures $\tilde{v}$ and $v$ on $\left(M_{t+1}, \mathbb{B}\left(M_{t+1}\right)\right)$, respectively. Since $\tilde{v}$ is absolutely continuous with respect to $v$, and both measures are $\sigma$-finite, there exists a Radon-Nikodym derivative $f\left(m_{t+1} \mid \tilde{\Theta}, h^{(t, 2)}, w_{t}\right)$ such that for any $\tilde{M} \in \mathbb{B}\left(M_{t+1}\right)$,

$$
\tilde{v}=\int_{\tilde{M}} f\left(m_{t+1} \mid \tilde{\Theta}, h^{(t, 2)}, w_{t}\right) v\left(d m_{t+1}\right) .
$$

Hence, we require that for $m_{t+1} \in \operatorname{supp}(v)$,

$$
f\left(\tilde{\Theta} \mid h^{(t+1,2)}\right)=f\left(m_{t+1} \mid \tilde{\Theta}, h^{(t, 2)}, w_{t}\right),
$$

where $h^{(t+1,2)}=\left(h^{(t, 2)}, w_{t}, m_{t+1}\right)$.

\section{References}

[1] Alonso, R. and N. Matouschek (2008), "Optimal delegation," The Review of Economic Studies 75, 259-293.

[2] Ambrus, A., E. M. Azevedo, and Y. Kamada (2013), "Hierarchical cheap talk," Theoretical Economics, 8, 233-261.

[3] Aumann, R. J. and S. Hart (2003), "Long cheap talk," Econometrica, 71, 1619-1660.

[4] Austen-Smith, D. and J. S. Banks (2000), "Cheap talk and burned money," Journal of Economic Theory, 91, 1-16.

[5] Benoit, J.-P. and V. Krishna (1985), "Finitely repeated games," Econometrica, 53, 905-922.

[6] Blume, A., O. Board, and K. Kawamura (2007), "Noisy talk," Theoretical Economics, $2,395-440$.

[7] Crawford, V. P. and J. Sobel (1982), "Strategic information transmission," Econometrica, 50, 1431-1451.

[8] Dessein, W. (2002), "Authority and communication in organizations," The Review of Economic Studies, 69, 811-838.

[9] Forges, F. and F. Koessler (2008), "Long persuasion games," Journal of Economic Theory, 143, 1-35.

[10] Golosov, M., V. Skreta, A. Tsyvinski, and A. Wilson (2014), “Dynamic strategic information transmission," Journal of Economic Theory, 151, 304-341. 
[11] Goltsman, M., J. Hörner, G. Pavlov, and F. Squintani (2009), “Mediation, arbitration and negotiation," Journal of Economic Theory, 144, 1397-1420.

[12] Hörner, J. and A. Skrzypacz (2016), "Selling information," Journal of Political Economy, 124, 1515-1562.

[13] Holmström, B. (1977), "On incentives and control in organizations," Ph.D. thesis, Stanford University.

[14] Ivanov, M. (2010), "Communication via a strategic mediator," Journal of Economic Theory, 145, 869-884.

[15] Ivanov, M. (2015), "Dynamic information revelation in cheap talk," The BE Journal of Theoretical Economics, 15, 251-275.

[16] Karamychev, V. and B. Visser (2016), “Optimal signaling with cheap talk and money burning," International Journal of Game Theory, forthcoming.

[17] Kartik, N. (2007), "A note on cheap talk and burned money," Journal of Economic Theory, 71, 1619-1660.

[18] Kartik, N. (2009), "Strategic communication with lying costs," The Review of Economic Studies, 76, 1359-1395.

[19] Kartik, N., M. Ottaviani, and F. Squintani (2007), "Credulity, lies, and costly talk," Journal of Economic theory, 134, 93-116.

[20] Kolotilin, A. and H. Li (2016), "Relational communication with transfer," mimeo.

[21] Krishna, V. and J. Morgan (2004), "The art of conversation: eliciting information from experts through multi-stage communication," Journal of Economic Theory, 117, 147-179.

[22] Krishna, V. and J. Morgan (2008), "Contracting for information under imperfect commitment," The RAND Journal of Economics, 39, 905-925.

[23] Mathis, J. (2008), "Full revelation of information in Sender-Receiver games of persuasion," Journal of Economic Theory, 143, 571-584.

[24] Melumad, N. and T. Shibano (1991), "Communication in settings with no transfers," The RAND Journal of Economics, 22, 173-198.

[25] Milgrom, P. and R. Weber (1985), "Distributional strategies for games with incomplete information," Mathematics of Operations Research, 10, 619-632.

[26] Seidmann, D. J. and E. Winter (1997), "Strategic information transmission with verifiable messages," Econometrica, 65, 163-169.

[27] Spence, M. (1973), “Job market signaling," The Quarterly Journal of Economics, 87, $35-374$. 